\title{
Cyclopalladated Compounds with Polyhalogenated Benzylphosphanes for the Mizoroki-Heck Reaction
}

\author{
Cristina López-Mosquera, ${ }^{[\mathrm{a}]}$ Arnald Grabulosa, ${ }^{*[\mathrm{a}, \mathrm{b}]}$ Mercè Rocamora, ${ }^{[\mathrm{a}]}$ Mercè Font-Bardia, ${ }^{[\mathrm{c}]}$ and \\ Guillermo Muller [a]
}

${ }^{[a]}$ Dr. C. López-Mosquera, Dr. A. Grabulosa, Dr. M. Rocamora, Dr. G. Muller Departament de Química Inorgànica i Orgànica, Secció de Química Inorgànica, Universitat de Barcelona, Martí i Franquès, 1-11, 08028, Barcelona, Spain

E-mail: arnald.grabulosa@qi.ub.es

${ }^{[b]}$ Dr. A. Grabulosa Institut de Nanociència i Nanotecnologia (IN2UB), Universitat de Barcelona, 08028, Barcelona, Spain

${ }^{[c]}$ Unitat de Difracció de RX, Centres Científics i Tecnològics de la Universitat de Barcelona (CCiTUB),

Solé i Sabarís 1-3, 08028, Barcelona, Spain 


\section{ABSTRACT:}

42

Nine partially halogenated benzylphosphanes ArXCH2PR2 (ArX = 3,6-dichlorophenyl, 3,644 difluorophenyl and 3,4,5-trifluorophenyl; $\mathrm{R}=\mathrm{Ph}, \mathrm{Cy}, \mathrm{iPr}$ ) have been prepared and reacted with 45 palladium acetate to obtain the cyclometallated dimers $[\mathrm{Pd}(\mu-\mathrm{OAc})(\kappa 2-\mathrm{C}, \mathrm{P}-\mathrm{ArXCH} 2 \mathrm{PR} 2)] 2$. The 46 acetate bridge has been exchanged by bromide using lithium bromide and the obtained dimers have been 47 thoroughly characterised. The dimers with the non-halogenated phosphanes PhCH2PR2 $(\mathrm{R}=\mathrm{Ph}, \mathrm{iPr})$ 48 have also been prepared. Treatment with norbornadiene in the presence of silver tetrafluoroborate has

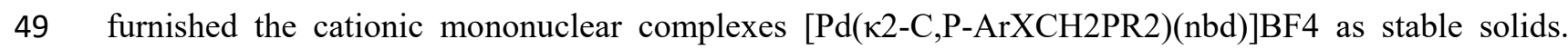
50 These complexes and some of the bromide dimers have been used as catalytic precursors in the 51 Mizoroki- Heck reaction between bromobenzene and butyl acrylate. The complexes efficiently catalyse 52 this transformation and important differences of activity are found depending on the ligand. In general, 53 fluorinated phosphanes give more active systems than chlorinated analogues. 
In 2010, Richard F. Heck, Ei-ichi Negishi and Akira Suzuki were awarded with the Nobel Prize in Chemistry "for palladium-catalyzed cross couplings in organic synthesis". The currently known as Mizoroki-Heck, Negishi and Suzuki-Miyaura reactions revolutionised the field of organic synthesis by allowing the formation of $\mathrm{C}-\mathrm{C}$ bonds under mild conditions.[1] The three reactions are efficiently catalysed by $\operatorname{Pd}(0)$ species and have been used to prepare countless molecules of industrial or pharmacological interest and remain central in the synthetic toolkit. The Mizoroki-Heck $[\mathrm{M}-\mathrm{H}]$ reaction is the coupling between an aryl halide or tosylate with an alkene, catalysed by $\operatorname{Pd}(0)$ in the presence of a base (Scheme 1).[2]

Compared to the Negishi or the Suzuki-Miyaura couplings, the $\mathrm{M}-\mathrm{H}$ reaction is particular because: 1) was the first to be discovered and paved the way to the discovery of the other cross-coupling reactions; 2) it is mechanistically distinct compared to the other cross-coupling reactions and 3) uses cheap and widely available unfunctionalised alkenes as coupling partners. The last reason is particularly important and explains the intense interest in the $\mathrm{M}-\mathrm{H}$ reaction in both academia and industry, a research effort that has given tens of thousands of publications and patents and has been collected in many reviews.[3] The generally accepted mechanism of the $\mathrm{M}-\mathrm{H}$ reaction involves a $\mathrm{Pd}(0) / \mathrm{Pd}(\mathrm{II})$ catalytic cycle[3d,3h] although usually the more stable $\operatorname{Pd}(\mathrm{II})$ systems are used as precatalysts. Simple palladium(II) salts such as palladium acetate or chloride,[2b,4] molecular complexes and organometallics[3b,5] as well as heterogeneous catalysts or "heterogenized" molecular species[6] have been used in the M-H reaction. Among the many systems studied, Herrmann's[4a,5,7] cyclometallated dimeric complexes with phosphanes (Figure 1) were found to produce exceptionally active systems for the reaction[8] and remain a landmark in the area.

These systems were discovered when exploring the use of o-tolylphosphanes in $\mathrm{M}-\mathrm{H}$ reactions, with palladium acetate as metallic precursor. Under catalytic conditions the methyl group of the o-tolyl substituent is activated forming the dimeric Herr mann's catalyst, with acetate bridges. Inspired by these results, a few years later Cole-Hamilton and co-workers[9] reported metallated complexes of palladium with simple benzylphosphanes (Figure 1), which were also found to be very active in $\mathrm{M}-\mathrm{H}$ reactions and also in Suzuki-Miyaura couplings. Cyclopalladated compounds with benzylphosphanes have indeed a long history. As early as 1975 Shaw and co-workers[10] showed that complexes trans-[PdC12L2] $(\mathrm{L}=$ $\mathrm{PBn} 2(\mathrm{tBu})$ and $\mathrm{PBn}(\mathrm{tBu} 2) 2)$ undergo internal metallation, with certain difficulty, to give complexes of the type $\left[\mathrm{Pd}(\mu-\mathrm{Cl})\left(\kappa 2-\mathrm{C}, \mathrm{P}-\mathrm{PBnRR} \mathrm{R}^{\prime}\right)\right] 2$. It was found that the bulkier $\mathrm{PBn}(\mathrm{tBu} 2) 2$ is metallated more readily than $\mathrm{PBn} 2(\mathrm{tBu})$. The bridging chlorides could be replaced by bromide or iodide and the bridges split by various ligands to give mononuclear species. A few years later, Vrieze and coworkers[11] reacted $\mathrm{PBnR} 2(\mathrm{R}=\mathrm{Cy}, \mathrm{tBu})$ with $[\mathrm{Rh}(\operatorname{cod}) \mathrm{Cl}] 2,[\operatorname{Ir}(\operatorname{cod}) \mathrm{Cl}] 2, \mathrm{PdCl} 2$ and $\mathrm{PtCl} 2$ (benzonitrile)2, 
92 obtaining the corresponding cyclometallated compounds. It was found that steric effects have a large 93 influence on the rates of the reactions. Hiraki and co-workers[12] were the first to cyclopalladate 94 benzyldiphenylphosphane with palladium acetate, giving the dimeric acetate-bridged complex, which 95 could be converted into the corresponding chloro-bridged analogue by a metathesis reaction with lithium 96 chloride. Much more recently, Leung and coworkers[13] demonstrated that steric shielding greatly 97 favours the palladation of benzylphosphanes and found that bis(tertbutyl)(diphenylmethyl)phosphane 98 readily palladates even under conditions known to disfavour the reaction. Interestingly, Gatineau et 99 al.[14] explored the cyclometallation of $\mathrm{PBn}(\mathrm{tBu})$-(o-tolyl) with palladium acetate and found that the o100 tolyl group not the benzyl, was palladated, proving that often $\mathrm{C}(\mathrm{sp} 3)-\mathrm{H}$ bonds are activated more easily 101 than C(sp2)-H. We reasoned that modification of the Cole-Hamilton systems by introduction of halogen 102 atoms in the ortho-metallated benzyl substituent would result in stronger $\mathrm{Pd}-\mathrm{Caryl}$ bonds and could give 103 more robust catalysts. In addition, we also envisaged the preparation of mononuclear, cationic versions 104 of the Cole-Hamilton's catalysts, stabilised by a norbornadiene to obtain more active catalysts, since the 105 dimeric systems are thought to give mononuclear complexes under catalytic conditions. The results on 106 the synthesis and characterisation of the polyhalogenated phosphanes and their derived cyclopalladated 107 compounds and the applications of the complexes in the $\mathrm{M}-\mathrm{H}$ reaction are described in this paper. 


\section{RESULTS AND DISCUSSION}

The cyclometallated dimers of Cole-Hamilton and co-workers[9] (Figure 1) were obtained by oxidative addition of o-bromobenzylphosphanes with the $\operatorname{Pd}(0)$ precursor [ $\mathrm{Pd} 2(\mathrm{dba}) 3]$. Although very efficient, this method requires a bromo substituent to be installed in the benzylphosphane, which would make the synthesis of the desired polyhalogenated phosphanes rather difficult. Therefore, the direct palladation of the polyhalogenated benzylphosphanes by $\mathrm{C}-\mathrm{H}$ activation with palladium acetate was studied, following a method used for simple benzylphosphanes.

\section{Benzylphosphanes}

The synthesis of di- and trihalogenated benzylphosphanes was explored by two methods (Scheme 2): the alkylation of secondary phosphanes with benzyl bromides in the presence of base (A)[15] and the treatment of chlorophosphanes with benzylic Grignard reagents (B).

Although both methods allowed the synthesis of the desired ligands, method B provided slightly higher yields and much better reproducibility and therefore was selected as default. As the free phosphanes turned out to be very air-sensitive, they were immediately either coordinated or protected with borane for storage and complete characterisation. For comparison purposes, non-halogenated phosphanes benzyldiphenylphosphane (4a) and benzyldiisopropylphosphane (4c) were also considered. Phosphane $4 \mathrm{a}$ is commercially available while $4 \mathrm{c}$ was prepared by method $\mathrm{B}$ employing commercially available benzylmagnesium chloride solution and following a literature procedure.[16] The free phosphanes were characterised by $31 \mathrm{P}\{1 \mathrm{H}\}$ NMR spectroscopy while their borane adducts were characterised by $31 \mathrm{P}\{1 \mathrm{H}\}, 1 \mathrm{H}$ and $13 \mathrm{C}\{1 \mathrm{H}\}$ NMR spectroscopy, IR spectroscopy, mass spectrometry and chemical analysis. The most relevant NMR data is given in Table 1. As expected, 31P chemical shifts strongly depended on the identity of the phosphane groups, with only a minor influence of the substitution in benzyl group. Diphenylphosphanes (a) appeared at the highest field followed by dicyclohexylphosphanes (b) and finally diisopropylphoshines (c). It was possible to grow crystals of phosphane-boranes $2 \mathrm{a}^{\prime}$ and $3 \mathrm{c}^{\prime}$ by slow diffusion of ethanol into concentrated dichloromethane solutions of the corresponding phosphane-boranes, at $4{ }^{\circ} \mathrm{C}$. The molecular structures are shown in Figure 2 along with a selection of bond lengths and angles.

No significant differences in bond lengths and angles were found for adducts $2 \mathrm{c}^{\prime}$ and $3 \mathrm{c}^{\prime}$, which show similar values to previously reported benzylphosphane-boranes.[14,19] 


\section{Neutral Palladium Complexes}

In the present work, with the aim of obtaining cyclometallated complexes the reaction of benzylphosphanes and palladium acetate was studied in detail in toluene due to increased stability of phosphanes in this solvent. Palladium acetate has been known to be particularly effective to palladate phosphanes.[12,14,20] When the reaction was performed at room temperature, palladium coordination complexes ([Pd(OAc)nP2]) were obtained and could be unequivocally identified upon conversion to trans-[PdBr2P2] by treatment with lithium bromide in acetone. An increase of the temperature was necessary to observe the $\mathrm{C}(\mathrm{sp} 2)-\mathrm{H}$ activation of the benzyl substituent of the phosphane. From temperatures in the range $50-60{ }^{\circ} \mathrm{C}$ it was already possible to detect cyclometallated complexes. The addition of a base like $\mathrm{NaOAc}$ was convenient to favour the $\mathrm{C}-\mathrm{H}$ activation and improve the yield of the palladium dimer and the reproducibility of the reaction.

Palladium dimers $[\mathrm{Pd}(\mu-\mathrm{OAc})(\kappa 2-\mathrm{PC})] 2(6 \mathrm{c}, 7 \mathrm{~b}, 8 \mathrm{a})$ containing the desired five-membered PdPC ring were obtained at $60{ }^{\circ} \mathrm{C}$ in $12 \mathrm{~h}$ while heating at $80^{\circ} \mathrm{C}$ allowed the formation of the cyclometallated dimers for all the phosphanes (Scheme 3).

The cyclopalladation of the benzylic phosphanes could be monitored by $31 \mathrm{P}\{1 \mathrm{H}\}$ NMR spectroscopy. In all cases different amounts (10-30 \%) of coordination compounds [Pd(OAc)nP2] were also detected as well as small amounts of starting palladium acetate and palladium black. The reaction mixture containing the palladium dimer was filtered through celite and the crude toluene solution was evaporated to dryness. The reaction of the solid residue with $\mathrm{LiBr}$ in acetone at room temperature for two hours led to the substitution of the acetate bridge and to the formation of the dimeric compounds $[\{\mathrm{Pd}(\kappa 2-\mathrm{PC})\}(\mu-\mathrm{Br})] 2(8,9,10$ and 12).[8b,12,14,20] In some occasions the coordination compound trans-[PdBr2P2] was also obtained and was separated by precipitation or column chromatography. In some cases, like with phosphane $2 b$ or if the reaction was carried out without adding sodium acetate, the formation of other compounds was observed (Scheme 4).

In this case the reaction of $2 \mathrm{~b}$ with palladium acetate yields the expected dimer $6 \mathrm{~b}$ but also the monomeric species $6 b^{\prime}$ resulting from the splitting of this dimer with another equivalent of $2 b$ and the coordination compound $[\mathrm{Pd}(2 \mathrm{~b}) 2(\mathrm{OAc}) 2]$. The ratio between the three species was 1.0:0.7:0.5 approximately. It could be observed that when mixtures of the metallated dimers and the corresponding coordination compounds $[\mathrm{Pd}(\mathrm{PR} 2 \mathrm{CH} 2 \mathrm{ArX}) 2(\mathrm{OAc}) 2]$ are left at $80{ }^{\circ} \mathrm{C}$ in the presence of Figure 3. $31 \mathrm{P}\{1 \mathrm{H}\}$ NMR spectrum of a mixture of brominated complexes obtained from $2 \mathrm{~b}$. Eur. J. Inorg. Chem. 0000, 0-0 www.eurjic.org 4 (C) 2020 Wiley-VCH Verlag GmbH \& Co. KGaA, Weinheim sodium acetate for long periods of time, the monomeric complexes $[\operatorname{PdBr}((\kappa 2-\mathrm{PC})(\mathrm{PR} 3)]]$ and unidentified decomposition products are obtained. 31P $\{1 \mathrm{H}\}$ NMR spectroscopy was an invaluable tool to analyse this kind of mixtures. As an example, Figure 3 shows the 31P $\{1 \mathrm{H}\}$ NMR spectrum of complexes of Scheme 4. 
Complexes 10 showed low solubility in common organic solvents such as dichloromethane, toluene or THF and all are stable as solids under nitrogen atmosphere. The new palladium complexes obtained $[\mathrm{Pd}(\kappa 2-\mathrm{PC})(\mu-\mathrm{Br})] 2(8,9,10,11$ and 12$)$ were characterized by elemental analysis, infrared spectroscopy and multi-nuclear $(31 \mathrm{P}\{1 \mathrm{H}\}, 1 \mathrm{H}, 19 \mathrm{~F}$ and $13 \mathrm{C}\{1 \mathrm{H}\})$ NMR spectroscopy. The metallated dimers with acetate bridge showed broad signals in both the $31 \mathrm{P}\{1 \mathrm{H}\}$ and $1 \mathrm{H}$ NMR spectra, in particular those with the diphenylphoshino group (a) and only the $31 \mathrm{P}\{1 \mathrm{H}\}$ spectra are presented. This is due to the fluxional character of the compounds and to the possible existence of an equilibrium between the monomer and the dimer, owing to the weakly bound acetate anions.[5] Relevant NMR data is summarized in Table 2.

As expected, 13P $\{1 \mathrm{H}\}$ NMR spectra showed a shift towards lower fields in all the complexes with respect to free phosphane. The ring contribution effect[21] is very important as reflected for example in the spectrum of complex 9b' (Table 3) with the same phosphane in the cyclometallated compound $(\delta=+71.5 \mathrm{ppm})$ and as simply P-coordinated ligand $(\delta=+28.4 \mathrm{ppm})$. The shift values followed the order -PiPr2 (c) > -PCy2 (b) $>-\mathrm{PPh} 2$ (a) for each benzyl group, and the sequence -2,5$\mathrm{F} 2 \mathrm{Ph}>-\mathrm{Ph}>-3,4,5-\mathrm{F} 3 \mathrm{Ph}>-2,5-\mathrm{C} 12 \mathrm{Ph}$ for each $-\mathrm{PR} 2$ moiety. Furthermore, the mixture of the two possible isomers (cis and trans) was observed in the acetate bridge complexes $5 \mathrm{~b}, 6 \mathrm{a}$ and $7 \mathrm{a}$, and in the bromide bridge for complexes $9 \mathrm{a}, 10 \mathrm{a}, 12 \mathrm{a}$ and $12 \mathrm{c}$.

${ }^{19} \mathrm{~F}\{1 \mathrm{H}\}, 13 \mathrm{C}\{1 \mathrm{H}\}$ and $1 \mathrm{H}$ NMR spectra of 5-12 showed less significant variations when forming the metallacycles. The values of the signals of the complexes bearing -PiPr2 and -PCy2 moieties are similar, in accordance with their comparable steric and electronic characteristics. It is interesting to note that the methyl groups of the isopropyl moiety in complexes derived from phosphanes $\mathrm{c}$ are not equivalent and appear as doublet of doublet sets.

Free phosphanes 2 containing the difluorobenzyl group showed a $4 \mathrm{JPF}$ of about $10 \mathrm{~Hz}$, however in the dimeric palladacycles 5 and 8 this coupling was not observed at the same $101.1 \mathrm{MHz}$ field. However, in the case of mononuclear complexes $6 b^{\prime}$ and $9 b^{\prime}$ (Table 3 ) the coupling constant reappears at the coordinated phosphane with values around $30 \mathrm{~Hz}$, but here there are two fluorine atoms in the same four bonds range 4JPF. In order to ascertain the origin of the P-F coupling the splitting of the dinuclear complex 9c with PCy3 was performed to obtain complex 13c (Scheme 5).

To a toluene solution of $9 \mathrm{c}$ a slight excess of tricyclohexylphosphane was added at room temperature, the complete splitting of the dimer was observed by $31 \mathrm{P}\{1 \mathrm{H}\}$ NMR after one hour of reaction. The neutral compound $13 \mathrm{c}$ was readily obtained and characterized by standard methods. The spectra were obtained in $\mathrm{CDCl} 3$ and it showed small amounts of the product of the $\mathrm{Pd}-\mathrm{Br} / \mathrm{Cl}$ halogen exchange, leading to the duplication of signals both in the $1 \mathrm{H}$ and $31 \mathrm{P}\{1 \mathrm{H}\} \mathrm{NMR}$. The $31 \mathrm{P}\{1 \mathrm{H}\} \mathrm{NMR}$

The 31P $\{1 \mathrm{H}\}$ NMR signals are consistent with the presence of only the trans isomer (2JPP = $414.0 \mathrm{~Hz}$ ) of 13c. Furthermore, the coupling between the phosphorus atom of the PCy3 and the fluorine atom in ortho position of the phenyl ligand was observed $(4 \mathrm{JPF}=27.4 \mathrm{~Hz})$. This means that the 4JPF 
observed in the mononuclear complexes $6 b^{\prime}$ and $9 b^{\prime}$ in the signal of the monodentate phosphane could be assigned to the same coupling with the fluorine in ortho position of the phenyl ligand. The exclusive formation of the trans isomer can be justified on steric arguments although electronic factors could also play a role.[22]

Despite many attempts, single crystals suitable for X-ray diffraction could not be obtained for any of complexes with the halogenated phosphanes. Fortunately, single crystals could be obtained for dimers $12 \mathrm{a}$ and $12 \mathrm{c}$, containing the simple benzylphosphanes $4 \mathrm{a}$ and $4 \mathrm{c}$ respectively. The structures of the dimers are shown in Figure 4.

The structures contain the expected dimeric complexes with square-planar geometries around the Pd atoms. Both structures correspond to the transoid isomers and have a crystallographic inversion centre and hence the $\{\mathrm{Pd} 2 \mathrm{Cl} 2\}$ moiety is completely flat. The cyclometallation forces a much smaller $\mathrm{P}-$ $\mathrm{Pd}-\mathrm{C} 1$ angle compared to the others around the metal centre. Interestingly, coordination bonds of $12 \mathrm{a}$ are slightly shorter than those of $12 \mathrm{c}$, probably due to less steric requirements of $4 \mathrm{a}$ compared to $4 \mathrm{c}$. Rather surprisingly, the structures of $12 \mathrm{a}$ and $12 \mathrm{c}$ are the first to be reported for bromo-bridged dimers with cyclometallated benzylphosphanes. Indeed, there are only a few structures of chloro-bridge dimers,[20,23] including chloro-analogue of 12a, described by Smoliakova and co-workers.[23] The parameters of $12 \mathrm{a}$ and $12 \mathrm{~b}$ are similar to those reported structures except the $\mathrm{Pd}-\mathrm{Br}$ distances which as expected are longer than the $\mathrm{Pd}-\mathrm{Cl}$.

\section{Ionic Palladium Complexes}

Reaction of a dichloromethane solution of the palladium dimers $8 \mathrm{c}, 9 \mathrm{c}, 10 \mathrm{a}-\mathrm{c}, 12 \mathrm{a}$ and $12 \mathrm{c}(1$ equiv.) with a slight excess of norbornadiene and silver tetrafluoroborate (3 equiv.) afforded the ionic

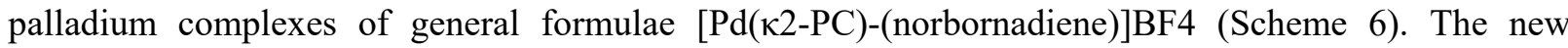
compounds were obtained as pure solids after separation of the silver bromide by filtration through a celite pad.

The ionic complexes were characterized by elemental analysis, infrared spectroscopy and multinuclear NMR spectroscopy. There are no significant changes in the NMR of the metallacycle (Table 4 and Figure 5).

Interestingly, the $1 \mathrm{H}$ NMR spectra shows the splitting of the signal of the methylene group of the norbornadiene ligand. In addition, the signals of the olefinic protons differ markedly due to the distinct ligands in trans position, phosphorus or carbon. The contacts observed in the NOESY spectrum of $15 \mathrm{c}$ (Figure 5) allowed to assign the signal at lower fields to the double bond trans to the phosphorus atom.

The molecular structure of $17 \mathrm{a}$ could be obtained by X-ray diffraction methods and it is represented in Figure 6. 
The molecular structure confirms the identity of $17 \mathrm{a}$ as a mononuclear complex with the $\mathrm{Pd}$ atom containing the cyclometallated ligand $4 \mathrm{a}$ and a norbornadiene coordinated by the two double bonds. The distances between the $\mathrm{Pd}$ atom and $4 \mathrm{a}$ are slightly longer than in 12a and the norbornadiene ligand is coordinated in slightly asymmetric fashion because the $\mathrm{C} 1-\mathrm{C} 2$ bond is closer to the $\mathrm{Pd}$ than the $\mathrm{C} 6-\mathrm{C} 7$ bond. To the best of our knowledge, the structure of $17 \mathrm{a}$ is the first one ever reported to contain a palladium coordinated to a metallated phosphane and a diene. In the reported structures of $[\mathrm{PdCl} 2(\mathrm{nbd})][24]$ and $[\mathrm{PdBr} 2(\mathrm{nbd})][25]$ the distances between the $\mathrm{Pd}$ and the diene are considerably shorter. In contrast, other structures containing the palladium embedded in a palladacyclopentadiene[26] present similar Pd-nbd distances than in 17a. This suggests that the cyclometallated phosphane exerts a strong $\sigma$-donation that weakens the bonds of $\mathrm{Pd}$ with the nbd in trans as does in palladacyclopentadienes.

\section{Mizoroki-Heck Reactions}

To test the performance of the cyclopalladated compounds, the $\mathrm{M}-\mathrm{H}$ reaction between bromobenzene and butyl acrylate (Scheme 7) was chosen.

The palladium complexes $9 \mathrm{c}$ and $15 \mathrm{c}$, containing the same phosphane ligand $2 \mathrm{c}$, were selected to establish the optimal reaction conditions. In order to study the stability of these complexes, some melting point determinations were carried out, but decomposition was found instead. The fluorinated neutral dimeric complex 9c decomposed to palladium black at approximately $225^{\circ} \mathrm{C}$ while the cationic counterpart $15 \mathrm{c}$ did the same at $165{ }^{\circ} \mathrm{C}$. For the non-fluorinated complexes $12 \mathrm{a}$ and $17 \mathrm{a}$, thedecomposition temperatures were approximately 215 and $160{ }^{\circ} \mathrm{C}$ respectively.

The initial catalytic experiments were carried out with $15 \mathrm{c}$ and sodium acetate as base. The ratio $\mathrm{Pd} / \mathrm{PhBr} /$ butyl acrylate was set to 1:1000:1500 (0.1\% catalyst loading) while the $\mathrm{PhBr} / \mathrm{NaOAc}$ ratio was set to $1: 1.1$. Under these conditions, no formation of the $\mathrm{M}-\mathrm{H}$ product was observed at $80{ }^{\circ} \mathrm{C}$, and the temperature was gradually increased in intervals of $10{ }^{\circ} \mathrm{C}$ until at $120{ }^{\circ} \mathrm{C}$ some product could be detected after $1 \mathrm{~h}$ of reaction time. Therefore, a compromise temperature of $130{ }^{\circ} \mathrm{C}$ was selected because it was high enough to have an active system, but well below the decomposition temperature of the complexes. In the literature, temperatures in the range $115-165^{\circ} \mathrm{C}$ are commonly used in $\mathrm{M}-\mathrm{H}$ reactions using cyclopalladated compounds as catalytic precursors.[27] were tested as a base. After $20 \mathrm{~h}$, the conversions using precursor $15 \mathrm{c}$ were 3,13 and $80 \%$ respectively so caesium carbonate was selected as by default base.

Then the effect of the amount of catalytic precursor was studied. When the relative amount of the palladium complex (15c) was duplicated to $0.2 \%(\mathrm{Pd} / \mathrm{PhBr} /$ butyl acrylate $=2: 1000: 1500)$, the rate of 
the reaction decreased, achieving a TOF value of $70 \mathrm{~h}-1$ at $1 \mathrm{~h}$, compared to $260 \mathrm{~h}-1$ with $\mathrm{Pd} / \mathrm{PhBr} /$ butyl acrylate $=1: 1000: 1500$. The rate reduction is significant because with $0.1 \%$ catalyst loading, the $50 \%$ conversion time was attained after approximately $3 \mathrm{~h}$, while doubling the loading of the precatalyst delayed this time until $4 \mathrm{~h}$. For this reason, the catalyst loading was set to $0.1 \%$. The use of an excess of olefin was also detrimental on the rate because with a $\mathrm{Pd} / \mathrm{PhBr} /$ butyl acrylate $=$ 1:1000:3000 the TOF was $110 \mathrm{~h}-1$. It seems, therefore, that the activity does not depend on the total amount of palladium. Evolution of the catalytic precursor to colloidal $\operatorname{Pd}(0)$ species could justify this behavior.[27,28]

The effect of the concentration was also studied. Halving the concentration from 0.67 to $0.38 \mathrm{M}$ led to similar conversions and TOF values. However, the reproducibility of the reaction was better at higher dilution, probably because the formation of palladium agglomerates is less favoured.

The cationic complex $15 \mathrm{c}$ showed a higher initial activity at $1 \mathrm{~h}(\mathrm{TOF}=260 \mathrm{~h}-1)$ than the neutral dimer $9 \mathrm{c}(\mathrm{TOF}=180 \mathrm{~h}-1)$ but at longer reaction times both precursors led to the same results. This may indicate the opening of dimer $9 \mathrm{c}$ forming active, mononuclear species over time.

After having optimised the reaction conditions, we performed the systematic screening of the

The conversions and selectivities (which were found to be $>95 \%$ towards the $\mathrm{M}-\mathrm{H}$ product) were determined by GC. In a few cases, Pd black could be observed in the reaction flasks after the consumption of the reagents. It was found that the precursors were active, giving good conversions in most cases towards the $\mathrm{M}-\mathrm{H}$ product after $6 \mathrm{~h}$ of reaction time. There were, however, important differences in reaction rates depending on the phosphane substituents, especially at $1 \mathrm{~h}$ reaction times. There is not a clear trend when comparing the performance between monomeric and dimeric precursors with the same ligand. In the case of ligand 2c (entries 1 and 5) and $4 a$ (entries 2 and 7) the differences are relatively minor suggesting the formation of the same mononuclear catalytically active species from dimers.[29] In contrast, monomeric complex 16c (entry 6) unexpectedly gives very low conversions and TOFs regardless of the reaction time while its dimeric counterpart 12c (entry 3 ) is one of the most active. These differences show that the ligand influences the active species formed under catalytic conditions. It seems that sometimes the monomeric and dimeric complexes lead to the same catalytically active species and sometimes not, or less efficiently.

In some cases, the activity of the precursors increases with the electronegativity of the substituents of the cyclometallated aryl ring. This can be clearly seen comparing entries $6(2 \mathrm{H}$ in the aryl $) 4(2 \mathrm{Cl})$ and 5 (2F) and may be due to the stronger Caryl-Pd bond in halogenated phosphanes, as originally reasoned when the halogenated benzylphosphanes were designed. This is however not general: ligand $3 \mathrm{c}$ (entry 10) gives a less active system than ligand 2c (entry 5) despite the former having a trifluorinated aryl ring compared to a difluorinated ring for the latter.

For the trifluorinated aryl ring it is found that the diphenylphosphanes produce less active precursors compared to diisopropyl or dicyclohexylphosphanes (entries 8-10), but in contrast the most 
329 active system overall, 17a (entry 7) is the simple benzyldiphenylphosphane, in accordance with the 330 results of Cole-Hamilton and co-workers.[9] With precursor 15c the M-H with styrene was also tested 331 (entry 11) giving very similar results than with butyl acrylate (entry 5).

332 In order to explore the usefulness of catalysts based on halogenated phosphanes in the $\mathrm{M}-\mathrm{H}$ of 333 other aryl bromides, a few experiments were also carried out with the reaction of butyl acrylate with 334 electron-poor 4-bromobenzaldehyde and with electron-rich 4-bromoanisol (Table 6). As expected, the reactions with 4-bromobenzaldehyde (entries 1-3) proceeded much faster than 336 with bromobenzene, due to the weaker nature of the $\mathrm{C}-\mathrm{Br}$ bond, which favours oxidative addition to $337 \operatorname{Pd}(0)$. In spite of this, the tendencies with this substrate were the same as those observed in Table 5. 338 Unsurprisingly, 4-bromoanisol (entry 4) was a poorer substrate than bromobenzene (Table 5, entry 5) 339 due to the stronger $\mathrm{C}-\mathrm{Br}$ bond. 
This paper has described the systematic preparation of new benzylphosphanes partially 344 halogenated at the phenyl group, showing that the reaction of benzylmagnesium reagents with 345 chlorophosphanes is the best method to prepare such compounds. The tendency of benzylphosphanes to 346 form cyclopalladated compounds has been used to prepare acetate-bridged and bromide-bridged complexes as pure stable solids. Bromide scavenging by silver tetrafluoroborate has allowed the obtention of cationic, nbd-stabilised mononuclear complexes. The use of $0.1 \%$ loading of palladium complexes in the Mizoroki- Heck reaction between bromobenzene and butyl acrylate has provided good conversions after a few hours for several systems. The results are in the same range that those obtained by other palladacycles[27,30] under similar conditions and confirm that cyclopalladated complexes are an excellent choice for $\mathrm{M}-\mathrm{H}$ reactions.[5,8a,8c,31]

Although the original idea that halogenated benzylphosphanes would give cyclopalladated compounds with stronger Pd-Caryl bonds and hence more robust and active precursors has been observed in a few cases, in general the halogenated precursors have not provided better systems than simple, nonhalogenated benzylphosphanes.

The palladated phosphane has an important influence in the catalytic outcome but so far it has not been possible to rationalise the results of individual catalytic precursors and find clear structure-activity relationships using simple parameters of the metallated phosphane. This stems from the deficient knowledge of the nature of the catalytically active species formed from cyclometallated compounds under M-H conditions despite many efforts.[4a,27,29,32] 
General Data: All compounds were prepared under a purified nitrogen atmosphere using standard Schlenk and vacuum-line techniques. The solvents were purified by a solvent purification system or by standard procedures[33] and kept under nitrogen. Unless otherwise noted, all reagents were purchased from commercial sources and were used without further purification. Benzyl bromides must be handled with care, manipulated in an efficient hood, wear protective gloves and eye protection because they may cause skin, eye and respiratory track irritation. $1 \mathrm{H}, 13 \mathrm{C}\{1 \mathrm{H}\}, 31 \mathrm{P}\{1 \mathrm{H}\}$ and $19 \mathrm{~F}$ NMR spectra were recorded at room temperature with 250, 300 and $400 \mathrm{MHz}$ spectrometers using $\mathrm{CDCl} 3$ as solvent unless otherwise specified. 1H-1H NOESY spectra were recorded in $500 \mathrm{MHz}$ spectrometers. Chemical shifts are reported in ppm relative to residual solvent peaks. IR spectra were recorded in $\mathrm{KBr}$ and the main absorption bands are expressed in $\mathrm{cm}-1$. Mass spectrometry (MS) and highresolution mass (HRMS) analyses were carried out in a time-of-flight instrument using electrospray ionisation. The microanalyses given are the best that could be obtained. The discrepancies observed are probably due to the presence of residual solvents (as observed by $1 \mathrm{H}$ NMR), which were impossible to remove despite leaving the complexes under high vacuum for several hours. Gas chromatography analyses of the catalytic runs were performed using a gas chromatograph, equipped with a FID detector, and a capillary column.

Synthesis of Phosphane-boranes: To a mixture of $1 \mathrm{mmol}$ of the appropriate benzyl bromide and 1.2 mmol of previously ground magnesium turnings, $5 \mathrm{~mL}$ of diethyl ether were added, and the mixture was stirred at room temperature for $1 \mathrm{~h}$. The reaction was moderately exothermic, and during the formation of the Grignard reagent, the solution turned to a dark grey colour. The solution was then cooled to $0{ }^{\circ} \mathrm{C}$ and $1 \mathrm{mmol}$ of ClPR2 in $10 \mathrm{~mL}$ of diethyl ether were added dropwise. The mixture was stirred for $1 \mathrm{~h}$, allowing the reaction to warm up to room temperature and $1.5 \mathrm{~mL}$ of boranedimethyl sulfide solution (1 $\mathrm{M}$ in THF) were added. $10 \mathrm{~mL}$ of a degassed solution of $10 \% \mathrm{NH} 4 \mathrm{Cl}$ was added and the mixture was stirred for $30 \mathrm{~min}$ to allow hydrolysis of the unreacted magnesium turnings. The diethyl ether was removed under reduced pressure and the resulting aqueous mixture was washed with dichloromethane (3 $\times 5 \mathrm{~mL}$ ). The organic layer was filtered and dried with anhydrous Na2SO4 and the resulting solution was concentrated to dryness. The crude product was purified as detailed for each comstirred for $30 \mathrm{~min}$ to allow hydrolysis of the unreacted magnesium turnings. The diethyl ether was removed under reduced pressure and the resulting aqueous mixture was washed with dichloromethane $(3 \times 5 \mathrm{~mL})$. The organic layer was filtered and dried with anhydrous $\mathrm{Na} 2 \mathrm{SO} 4$ and the resulting solution was concentrated to dryness. The crude product was purified as detailed for each com pound under vacuum to half its volume, and absolute ethanol was added until appearance of turbidity. Storing the mixture at $-20{ }^{\circ} \mathrm{C}$ for $24 \mathrm{~h}$ resulted in the precipitation of the phosphane-borane adduct, which was filtered off and dried under vacuum. 
(2,5-Dichlorobenzyl)diphenylphosphane-borane (1a'): The preparation was carried out according to the general procedure. The crude product was purified by column chromatography (hexane/ethyl acetate mixtures) to obtain the phosphane-borane as a colourless resin $(0.247 \mathrm{~g}, 69 \%$ yield). 1H NMR: 3.77 (d, $2 \mathrm{H} ; \mathrm{J}=12.0 \mathrm{~Hz}, \mathrm{CH} 2(\mathrm{Bn}))$; 6.90-7.10 (m, 3H, CH(Ar)); 7.30-7.60 (m, 10H, CH(Ph)). 13C $\{1 \mathrm{H}\} \mathrm{NMR}$ : $30.8(\mathrm{~d}, \mathrm{~J}=31.6 \mathrm{~Hz}, \mathrm{CH} 2(\mathrm{Bn})) ; 128.6(\mathrm{~s}, \mathrm{CH}) ; 128.8(\mathrm{~d}, \mathrm{~J}=9.6 \mathrm{~Hz}, \mathrm{CH}) ; 130.5$ (s, CH); 131.7 (s, CH); $131.8(\mathrm{~d}, \mathrm{~J}=3.2 \mathrm{~Hz}, \mathrm{CH}) ; 132.2(\mathrm{~m}, \mathrm{C}) ; 132.3$ (d, J = 3.8 Hz, C); 132.6 (m, C); 132.8 (d, J = $8.9 \mathrm{~Hz}$, $\mathrm{CH}) ; 133.1(\mathrm{~d}, \mathrm{~J}=5.7 \mathrm{~Hz}, \mathrm{C}) .31 \mathrm{P}\{1 \mathrm{H}\}$ NMR: 17.9 (d, J = 55.7 Hz). IR: 3084, 3058, 2924, 2395, 2361, $2341 v(\mathrm{~B}-\mathrm{H}), 1640,1619,1431,1099,1053,742,690$. HRMS: 355.0385 [M-3H]+.

Dicyclohexyl(2,5-dichlorobenzyl)phosphane-borane (1 $\left.\mathbf{b}^{\prime}\right)$ : The preparation of this compound was carried out according to the general procedure and was purified by recrystallisation in dichloromethane/pentane, affording the phosphane-borane as a white powder $(0.181 \mathrm{~g}, 49 \% \mathrm{yield}) .1 \mathrm{H}$ $\operatorname{NMR}(-0.03)-0.80$ (br; 3H; J $\approx 10 \mathrm{~Hz}$; BH3); 1.10-1.90 (m; $22 \mathrm{H} ; \mathrm{CH}(\mathrm{Cy})+\mathrm{CH} 2(\mathrm{Cy})$ ); 3.16 (d; 2H; J = 11.9 Hz; CH2(Bn)); 7.14 (d; 1H; J = 8.7 Hz; CH(Ar)); 7.27 (d; 1H; J = 8.7 Hz; CH(Ar)); 7.52 (m; 1H; CH(Ar)). 13C $\{1 \mathrm{H}\}$ NMR: $24.5(\mathrm{~d}, \mathrm{~J}=26.8 \mathrm{~Hz} ; \mathrm{CH} 2(\mathrm{Bn})) ; 26.0(\mathrm{~s} ; \mathrm{CH} 2) ; 26.9$ (m; CH2); 27.0 (d; J = $1.5 \mathrm{~Hz} ; \mathrm{CH} 2) ; 27.1$ (d; J = 1.9 Hz; CH2); 32.2 (d; J = 30.6 Hz; CH); 128.4 (d; J = 2.3 Hz; CH); 130.5 (d, $\mathrm{J}=1.5 \mathrm{~Hz} ; \mathrm{CH}) ; 132.2(\mathrm{~d}, \mathrm{~J}=4.5 \mathrm{~Hz} ; \mathrm{C}) ; 132.5(\mathrm{~d}, \mathrm{~J}=2.3 \mathrm{~Hz} ; \mathrm{CH}) ; 132.7$ (d; J = 2.5 Hz; C); 134.1 (d; J $=3.8 \mathrm{~Hz} ; \mathrm{C}) .31 \mathrm{P}\{1 \mathrm{H}\}$ NMR: 29.7 (m). IR: 3081, 3061, 2927, 2854, 2384, 2375, $2331 v(\mathrm{~B}-\mathrm{H}), 1465$, 1448, 1096, 1038, 893, 817. Anal. Calcd for C19H30BCl2P C $61.49 \%$, H $8.15 \%$; found C $61.48 \%$, H $9.15 \%$. HRMS: $367.1312[\mathrm{M}-3 \mathrm{H}]+, 369.1373[\mathrm{M}-\mathrm{H}]+$.

(2,5-Dichlorobenzyl)diisopropylphosphane-borane $\left(\mathbf{1}^{\prime}\right)$ : The preparation of this compound was carried out according to the general procedure. The crude product was recrystallised from dichloromethane/ ethanol to obtain the phosphane-borane as a white powder $(0.198 \mathrm{~g}, 68 \%$ yield $)$. $1 \mathrm{H}$ NMR: $0.00-0.80$ (br q; 3H; J $\approx 98 \mathrm{~Hz} ; \mathrm{BH} 3$ ); 1.06 (dd; 6H; J = 14.0, 7.2 Hz; $2 \times \mathrm{CH} 3(\mathrm{iPr})$ ); 1.11 (dd; $6 \mathrm{H} ; \mathrm{J}=14.4,7.2 \mathrm{~Hz} ; 2 \times \mathrm{CH} 3(\mathrm{iPr})) ; 2.01$ (dht; 2H; J = 10.0, 7.2 Hz; CH(iPr)); 3.11 (d; 2H; J = $12.0 \mathrm{~Hz}$; $\mathrm{CH} 2(\mathrm{Bn})) ; 7.08$ (m; 1H; CH(Ar)); $7.20(\mathrm{~m} ; 1 \mathrm{H} ; \mathrm{CH}(\mathrm{Ar})) ; 7.50$ (m;1H; $\mathrm{CH}(\mathrm{Ar}))$. 13C $\{1 \mathrm{H}\}$ NMR: 17.2 (d; J = 6.9 Hz; CH3); 22.4 (d; J = 30.6 Hz; CH2(Bn)); 24.3 (d; J = 26.8 Hz; CH); 128.5 (s; CH); 130.6 (s; CH); 132.1 (d; J = 5.4 Hz; C); $132.3(\mathrm{~s} ; \mathrm{CH}) ; 132.7$ (s; C); 133.8 (d; J=3.8 Hz; C). 31P\{1H\} NMR: 37.5 (q; J = 58.8 Hz). IR: 3084, 2965, 2930, 2875), 2375, 2355, 2334 v(B-H), 1471, 1454, 1096, 1038, 884, 820, 803. Anal. Calcd for C13H22BCl2P C $53.66 \%$, H $7.62 \%$; found C $51.49 \%$, H $8.12 \%$. HRMS: $287.0688[\mathrm{M}-3 \mathrm{H}]+, 289.0708[\mathrm{M}-\mathrm{H}]+$.

Diphenyl(2,5-difluorobenzyl)phosphane-borane (2a'): The preparation of this compound was carried out according to the general procedure and was purified by recrystallisation in dichloromethane/pentane, affording the phosphane-borane as a white powder $(0.117 \mathrm{~g}, 36 \%$ yield). $1 \mathrm{H}$ NMR: $0.40-1.30$ (br q; $3 \mathrm{H} ; \mathrm{J} \approx 96 \mathrm{~Hz} ; \mathrm{BH} 3) ; 3.53$ (d; 2H; J = 11.6 Hz; CH2(Bn)); 6.70-6.80 (m; 3H; CH(Ar)); 7.35-7.38 (m; 
4H; $\mathrm{CH}(\mathrm{Ar}))$; 7.39-7.44 (m; 2H; $\mathrm{CH}(\mathrm{Ar})$ ); 7.54-7.61 (m; 4H; CH(Ar)). 13C $\{1 \mathrm{H}\} \mathrm{NMR}: 26.8$ (d; J = $32.8 \mathrm{~Hz} ; \mathrm{CH} 2(\mathrm{Bn})) ; 115.4$ (dd; J = 23.8, $8.3 \mathrm{~Hz} ; \mathrm{CH}) ; 116.1$ (dd; J = 25.7, 8.9 Hz; CH); 118.4 (d; J = $25.0 \mathrm{~Hz} ; \mathrm{CH}) ; 121.3$ (m; C); 128.2 (d; J = 54.1 Hz; C); 128.9 (d; J = 9.6 Hz; CH); 131.6 (s; CH); 132.6 $(\mathrm{d} ; \mathrm{J}=9.0 \mathrm{~Hz} ; \mathrm{CH}) ; 156.9$ (d; J = 240.1 Hz; CF); 158.1 (d; J = 242.7 Hz; CF). 31P \{1H\} NMR: 17.7 (d; J = 71.6 Hz). 19F NMR: -121.4 (m; 1Fo); -118.6 (m; 1Fm). IR: 3078, 3052, 2962, 2921, 2406, 2398, $2352 v(\mathrm{~B}-\mathrm{H}), 1637,1614,1433,1096,1050,742,692$. Anal. Calcd for C19H18BF2P C $69.97 \%$, H $5.56 \%$; found C $68.30 \%$, H $5.76 \%$. HRMS: $323.1000[\mathrm{M}-3 \mathrm{H}]+, 329.0910[\mathrm{M}-(\mathrm{BH} 3)+(\mathrm{OH})]+$.

Dicyclohexyl(2,5-difluorobenzyl)phosphane-borane (2b'): The preparation of this compound was carried out according to the general procedure and was purified by recrystallisation in dichloromethane/pentane, affording the phosphane-borane as a white powder $(0.230 \mathrm{~g}, 68 \%$ yield $) .1 \mathrm{H}$ NMR: 0.00-0.80 (br q; 3H; J $\approx 74$ Hz; BH3); 1.10-1.90 (m; $22 \mathrm{H} ; \mathrm{CH}(\mathrm{Cy})+\mathrm{CH} 2(\mathrm{Cy})$ ); 3.00 (d; 2H; J = $11.2 \mathrm{~Hz} ; \mathrm{CH} 2(\mathrm{Bn})) ; 6.89$ (m; 1H; $\mathrm{CH}(\mathrm{Ar})) ; 6.98$ (m; 1H; $\mathrm{CH}(\mathrm{Ar})) ; 7.13(\mathrm{~m} ; 1 \mathrm{H} ; \mathrm{CH}(\mathrm{Ar})) .13 \mathrm{C}\{1 \mathrm{H}\}$ NMR: 20.3 (d; J = 27.5 Hz; CH2(Bn)); 26.0 (m; CH2); 26.8-27.1 (m; $2 \times \mathrm{CH} 2) ; 32.2$ (d; J = 30.6 Hz; $\mathrm{CH}) ; 115.1(\mathrm{dd} ; \mathrm{J}=7.64,22.9 \mathrm{~Hz} ; \mathrm{CH}) ; 116.2(\mathrm{dd} ; \mathrm{J}=8.4,25.2 \mathrm{~Hz} ; \mathrm{CH}) ; 118.8(\mathrm{~d} ; \mathrm{J}=24.5 \mathrm{~Hz} ; \mathrm{CH})$; $123.0(\mathrm{~m} ; \mathrm{C}) ; 156.8$ (d; J = 236.0 Hz; CF); 158.3 (d; J = 243.0 Hz; CF). 31P\{1H\} NMR: 30.0 (d; J = 67.0 Hz). 19F NMR: -123.1 (m; 1Fo); -118.6 (m; 1Fm). IR: 3073, 2927, 2849, 2363, 2337 v(B-H), 1497, 1451, 1207, 1064, 876, 809. Anal. Calcd for C19H30BF2P C 67.47 \%, H 8.94 \%; found C 67.19 \%, H $9.87 \%$. HRMS: 335.1903 [M-3H]+.

(2,5-Difluorobenzyl)diisopropylphosphane-borane $\left(\mathbf{2} \mathbf{c}^{\prime}\right)$ : The preparation of this compound was carried out according to the general procedure. The crude product was purified by column chromatography (hexane/ethyl acetate mixtures) to obtain the phosphane- borane as a white powder (0.157 g, 61 \% yield). 1H NMR: 0.00-0.80 (br q; 3H; J $\approx 86 \mathrm{~Hz}$; BH3); 1.15 (dd; 6H; J = 7.2, $1.6 \mathrm{~Hz}$; CH3(iPr)); 1.19 (dd; 6H; J = 7.2, 1.6 Hz; CH3(iPr)); 2.04 (dht; 2H; J = 10.8, 7.2 Hz; CH(iPr)); 3.03 (d; $2 \mathrm{H} ; \mathrm{J}=11.2 \mathrm{~Hz} ; \mathrm{CH} 2(\mathrm{Bn})) ; 6.86-6.94(\mathrm{~m} ; 1 \mathrm{H} ; \mathrm{CH}(\mathrm{Ar})) ; 6.95-7.20$ (m; 1H; $\mathrm{CH}(\mathrm{Ar})) ; 7.14-7.20$ (m; 1H; $\mathrm{CH}(\mathrm{Ar})) .13 \mathrm{C}\{1 \mathrm{H}\}$ NMR: $17.0(\mathrm{~d} ; \mathrm{J}=4.6 \mathrm{~Hz}$; CH3); 20.3 (d; J = 27.6 Hz; CH2(Bn)); 22.2 (d; J = $31.5 \mathrm{~Hz} ; \mathrm{CH}$ ); 115.2 (ddd; J = 3.1, 8.3, $23.8 \mathrm{~Hz} ; \mathrm{CH}$ ); 116.3 (ddd; J = 2.3, 9.1, $25.3 \mathrm{~Hz} ; \mathrm{CH}$ ); 118.8 (dt; $\mathrm{J}=3.0,24.5 \mathrm{~Hz} ; \mathrm{CH}) ; 122.8(\mathrm{~m} ; \mathrm{C}) ; 156.8(\mathrm{~d} ; \mathrm{J}=240.0 \mathrm{~Hz} ; \mathrm{CF}) ; 158.4(\mathrm{~d} ; \mathrm{J}=243.0 \mathrm{~Hz} ; \mathrm{CF}) .31 \mathrm{P}\{1 \mathrm{H}\}$ NMR: 36.1 (q, J = 58.5 Hz). 19F NMR:-122.9 (m; 1Fo); -118.6 (m; 1Fm). IR: 3090, 3070, 2979, 2962, 2936, 2875, 2378, 2346, $2369 v(\mathrm{~B}-\mathrm{H}), 1503,1463,1213,1192,1067,1038,870,820$. Anal. Calcd for C13H22BF2P C $60.50 \%$, H $8.59 \%$; found C $60.35 \%$, H $8.80 \%$. HRMS: 255.1287 [M-3H]+.

Diphenyl(3,4,5-trifluorobenzyl)phosphane-borane (3a'): The preparation of this compound was carried out according to the general procedure. The crude product was purified by column chromatography (hexane/ethyl acetate mixtures) to obtain the phosphane-borane as a white powder (0.182 g, $53 \%$ yield). 1H NMR: 0.40-1.40 (br q; 3H; J $\approx 112 \mathrm{~Hz} ; \mathrm{BH} 3$ ); 3.50 (d; 2H; J = $11.6 \mathrm{~Hz}$; 
$\mathrm{CH} 2(\mathrm{Bn})) ; 6.56(\mathrm{~m} ; 2 \times \mathrm{CH}(\mathrm{Ar})) ; 7.40-7.80(\mathrm{~m} ; 10 \mathrm{H} ; \mathrm{CH}(\mathrm{Ph})) .13 \mathrm{C}\{1 \mathrm{H}\} \mathrm{NMR}: 17.0(\mathrm{~d} ; \mathrm{J}=4.6 \mathrm{~Hz}$;

CH3); 20.3 (d; J = 27.6 Hz; CH2(Bn)); 22.2 (d; J = 31.5 Hz; CH); 115.2 (ddd; J = 3.1, 8.3, 23.8 Hz; $\mathrm{CH}) ; 116.3(\mathrm{ddd} ; \mathrm{J}=2.3,9.1,25.3 \mathrm{~Hz} ; \mathrm{CH}) ; 118.8(\mathrm{dt} ; \mathrm{J}=3.0,24.5 \mathrm{~Hz} ; \mathrm{CH}) ; 122.8(\mathrm{~m} ; \mathrm{C}) ; 156.8(\mathrm{~d} ; \mathrm{J}$ $=240.0 \mathrm{~Hz} ; \mathrm{CF}) ; 158.4$ (d; J = 243.0 Hz; CF). 31P $\{1 \mathrm{H}\}$ NMR: 17.2 (d; J = 62.9 Hz). 19F NMR: -162.1 (tdd; J = 19.9; 13.2; 5.3 Hz; 1Fp); -134.4 (dd; J = 21.0; 8.5 Hz; 2Fm). IR: 3096, 3058, 2916, 2378, 2343 $v(\mathrm{~B}-\mathrm{H}), 1622,1532,1451,1442,1349,1041,861,742,707,690$. Anal. Calcd for C19H17BF3P C $66.32 \%, \mathrm{H} 4.98 \%$; found C $65.30 \%$, H $5.34 \%$. HRMS: 341.0874 [M-3H]+, 347.0812 [M$(\mathrm{BH} 3)+(\mathrm{OH})]+$.

Dicyclohexyl(3,4,5-trifluorobenzyl)phosphane-borane $\left(3 b^{\prime}\right)$ : The preparation of this compound was carried out according to the general procedure. The crude product was purified by column chromatography (hexane/ethyl acetate mixtures) to obtain the phosphane-borane as a white powder (0.085 g, 24 \% yield). 1H NMR: 0.00-0.80 (br q; 3H; J $\approx 112 \mathrm{~Hz}$; BH3); 1.10-1.90 (m; $22 \mathrm{H}$; CH(Cy) + $\mathrm{CH} 2(\mathrm{Cy})) ; 2.94(\mathrm{~d} ; 2 \mathrm{H} ; \mathrm{J}=11.6 \mathrm{~Hz} ; \mathrm{CH} 2(\mathrm{Bn})) ; 6.92$ (t; 2H; J = 6.8 Hz; CH(Ar)). 13C $\{1 \mathrm{H}\} \mathrm{NMR}: 26.0$ (s; CH2); 26.9 (m; $2 \times$ CH2); 27.5 (d; J = 27.6 Hz; CH2(Bn)); 32.0 (d; J = 29.8 Hz; CH2); 114.2 (d; J = 16.9 Hz; CH); 130.3 (m; C); 138.9 (d; J = 254.0 Hz; CF); 151.0 (d; J = 254.0 Hz; CF). 31P \{1H\} NMR: 27.7 (d, J = 58.8 Hz). 19F NMR: -162.2 (td; J = 21.0, 3.7 Hz; 1Fp); -133.8 (dd; J = 19.9, 7.9 Hz; 2Fm). IR: 2933, 2857, 2375, 2343 v(B-H), 1530, 1445, 1035, 870, 797. Anal. Calcd for C19H29BF3P C 64.06 \%, H $8.21 \%$; found C $63.76 \%$, H $8.94 \%$. HRMS: 353.1812 [M-3H]+, 711.4023 [2M-H]+.

Diisopropyl(3,4,5-trifluorobenzyl)phosphane-borane $\left(\mathbf{3 c}^{\prime}\right)$ : The preparation of this compound was carried out according to the general procedure. The crude product was first subjected to column chromatography (hexane/ethyl acetate mixtures) and subsequently recrystallized from a mixture of dichloromethane/heptane to obtain the phosphane-borane as a white powder $(0.116 \mathrm{~g}, 42 \%$ yield). $1 \mathrm{H}$ NMR: 0.00-0.80 (br q; 3H; J $\approx 90.4$ Hz; BH3); 1.15 (dd; 6H; J = 7.2, 1.6 Hz; CH3(iPr)); 1.18 (dd; 6H; J = 7.2, $2.0 \mathrm{~Hz} ; \mathrm{CH} 3(\mathrm{iPr})$ ); 1.98 (dht; 2H; J = 10.4, 7.2 Hz; CH(iPr)); 2.95 (d; 2H; J = 11.6 Hz; CH2(Bn)); 6.94 (td; 2H; J = 6.8, 1.8 Hz; CH(Ar)). 13C $\{1 \mathrm{H}\}$ NMR: 17.1 (s; CH3); 22.0 (d; J = 31.4 Hz; CH2(Bn)); 27.6 (d; J = 26.9 Hz; CH); $114.2(\mathrm{~m} ; \mathrm{CH}) ; 130.0$ (s; C); 138.9 (dt; J = 12.8, 251.0 Hz; CF); 151 (dd; J = 10.9, 251.0 Hz; CF). 31P $\{1 \mathrm{H}\}$ NMR: 34.6 (q; J = 51.2 Hz). 19F NMR: -162.3 (td; J = 20.3; $4.1 \mathrm{~Hz}$; 1Fp); -133.8 (dd; J = 19.9; 7.9 Hz; 2Fm). IR: 3078, 3061, 3040, 2962, 2933, 2872, 2375, 2340, 2265 $v(\mathrm{~B}-\mathrm{H}), 1622,1588,1535,1465,1448,1349,1070,1038,861$. Anal. Calcd for C13H21BF3P C 56.56 \%, H $7.67 \%$; found C $56.00 \%$, H $8.35 \%$. HRMS: 273.1193 [M-3H]+.

Synthesis of Palladium Complexes: Cyclometallated palladium dimeric complexes with a bromide bridge were obtained by reaction of the corresponding phosphane with $\mathrm{Pd}(\mathrm{OAc}) 2$ followed by substitution of acetate by bromide. Only the preparation of $1 \mathrm{a} / 5 \mathrm{a} / \mathrm{ga}$ is described in detail and the rest of the complexes were obtained by the same protocol. Due to the easy oxidation of the phosphanes all 
operations must be carried out under nitrogen. The final purification of the cyclometallated dimer is performed by recrystallization or column chromatography (DCM/hexane).

\section{Di- $\mu$-bromo-bis $\{2-[($ diphenylphosphano)methyl]-3,6-dichlorophenyl-C1,P\}dipalladium(II) (8a)}

Preparation of Phosphane 1a: To a solution of $0.38 \mathrm{~g}(1.60 \mathrm{mmol})$ of 2,5-dichlorobenzyl bromide in $10 \mathrm{~mL}$ of diethyl ether $0.045 \mathrm{~g}(1.80 \mathrm{mmol})$ of previously ground magnesium turnings were added. The mixture was stirred at $20^{\circ} \mathrm{C}$ for $1 \mathrm{~h}$ avoiding any temperature increase. The dark grey solution was cooled to $0{ }^{\circ} \mathrm{C}$ and $0.330 \mathrm{~g}(1.5 \mathrm{mmol})$ of $\mathrm{ClPPh} 2$ in $5 \mathrm{~mL}$ of diethyl ether were added dropwise. The mixture was stirred for $1 \mathrm{~h}$, allowing the reaction to warm up to room temperature. The solvent was removed under reduced pressure giving a crude resin, which was dissolved in $10 \mathrm{~mL}$ of toluene and washed with deoxygenated water. The organic layer was separated and dried with anhydrous $\mathrm{Na} 2 \mathrm{SO} 4$ and filtered off. The solution of $1 \mathrm{a}(\delta \mathrm{P}=-12.8 \mathrm{ppm})$ was used immediately without further purification.

Preparation of Palladium Complexes 5a and 8a: The obtained phosphane solution was slowly added over a suspension of palladium acetate $0.314 \mathrm{~g}(1.40 \mathrm{mmol})$ and sodium acetate $0.254 \mathrm{~g}(1.80 \mathrm{mmol})$ in $10 \mathrm{~mL}$ of toluene at room temperature. After 5 min of stirring, the reaction mixture was heated at $80{ }^{\circ} \mathrm{C}$ for $12 \mathrm{~h}$. The disappearance of the free phosphane was monitored by $31 \mathrm{P}\{1 \mathrm{H}\} \mathrm{NMR}$. The solution was filtered, and the solvent removed under reduced pressure, giving crude $5 \mathrm{a}(\delta \mathrm{P}=+48.4 \mathrm{ppm})$. This crude and lithium bromide $0.155 \mathrm{~g},(1.80 \mathrm{mmol})$ were dissolved in acetone $(10 \mathrm{~mL})$ and stirred for $2 \mathrm{~h}$ at room temperature. The solvent was removed under reduced pressure, the solid dissolved in dichloromethane $(10 \mathrm{~mL})$ and washed several times with water. The organic phase was dried with anhydrous Na2SO4, filtered and after reducing the volume of dichloromethane, absolute ethanol was added. After several hours in the freezer the resulting yellow complex 8a was filtered off. The yield of calculated from the initial Pd(OAc)2: 0.30 g (40 \% yield). 1H NMR: 4.09 (d; 4H; J = 11.6 Hz; CH2(Bn)); 6.80-6.90 (m; 4H; CH(Ar)); 7.30-7.80 (m; 20H; CH(Ph)). 13C $\{1 \mathrm{H}\}$ NMR: 43.0 (d; J = 36.8 Hz; CH2(Bn)); 126.8 (s; CH); 128.8 (s; CH); 128.9 (d; J = 5.3 Hz; CH); 129.5 (s; C); 130.0 (s; C); 131.9 (s; CH); 133.4 (d; J = 11.5 Hz; CH); 139.8 (s; C). 31P\{1H\} NMR: 53.1 (s). IR: 3077, 3055, 2950, 2925, 2847, 1433, 1419, 1381, 1165, 1148, 1123, 1104, 1051. Anal. Calc. for C38H28Br2C14P2Pd2 C $43.02 \%$, H $2.66 \%$; found C $44.12 \%$ H $2.99 \%$. HRMS: $525.8080[(\mathrm{M} / 2)-2 \mathrm{H}]+$.

\section{Di- $\mu$-bromo-bis $\{2-[($ dicyclohexylphosphano)methyl]-3,6-dichlorophenyl-C1,P\}dipalladium(II)}

(8b): The procedure was analogous to that employed for 8a but using 1 b. The compound was obtained as a yellow solid after column chromatography. The yield was calculated relative to the initial $\mathrm{Pd}(\mathrm{OAc}) 2: 0.227 \mathrm{~g}(30 \%) . \delta \mathrm{P}(1 \mathrm{~b})=+2.6 \mathrm{ppm}(\mathrm{s}), \delta \mathrm{P}(5 \mathrm{~b})=65.3(\mathrm{~s}), 66.3(\mathrm{~s}) \mathrm{ppm} ; 1: 3$ ratio. $1 \mathrm{H}$ NMR: 0.80-2.60 (m; $44 \mathrm{H} ; \mathrm{CH}(\mathrm{Cy})+\mathrm{CH} 2(\mathrm{Cy})) ; 3.31$ (d; 4H; J = 10.4 Hz; CH2(Bn)); 6.88 (d; 2H; J = 8.4 Hz; $\mathrm{CH}(\mathrm{Ar})) ; 6.96(\mathrm{~d} ; 2 \mathrm{H} ; \mathrm{J}=8.7 \mathrm{~Hz} ; \mathrm{CH}(\mathrm{Ar})) .13 \mathrm{C}\{1 \mathrm{H}\} \mathrm{NMR}: 25.8(\mathrm{~s} ; \mathrm{CH} 2) ; 26.0(\mathrm{~d} ; \mathrm{J}=13.7 \mathrm{~Hz} ; \mathrm{CH})$; 
26.6 (d; J = 11.5 Hz; CH2); 28.2 (s; CH2); 28.9 (s; CH2); 34.4 (d; J = 34.5 Hz; CH2); 35.3 (d; J = 24.5 Hz; CH2(Bn)); 126.2 (s; CH); 127.3 (d; J = 24.5 Hz; C); 128.7 (s; CH); 139.9 (s; C); 144.4 (d; J = 16.0 Hz; C); 152.9 (s; C). 31P \{1H\} NMR: 78.7 (s). IR: 3046, 2930, 2850, 2784, 1446, 1419, 1157, 1047 , 851, 798. Anal. Calc. for C38H52Br2C14P2Pd2 C $42.06 \%$, H $4.83 \%$; found C $44.04 \%$, H $5.01 \%$. HRMS: $461.0183[(\mathrm{M} / 2)-\mathrm{Br}]+; 502.0452[(\mathrm{M} / 2)-\mathrm{Br}+(\mathrm{CH} 3 \mathrm{CN})]+, 1000.9545[\mathrm{M}-\mathrm{Br}]+$.

\section{Di- $\mu$-bromo-bis $\{2-[($ diisopropylphosphano)methyl]-3,6-dichlorophenyl-C1,P $\}$ dipalladium(II) (8c):}

The procedure was analogous to that employed for $8 \mathrm{a}$ but using $1 \mathrm{c}$. The compound was obtained as a yellow solid after column chromatography. The yield was calculated relative to the initial $\mathrm{Pd}(\mathrm{OAc}) 2$ : $0.168 \mathrm{~g}(26 \%) . \delta \mathrm{P}(1 \mathrm{c})=+10.1 \mathrm{ppm}(\mathrm{s}), \delta \mathrm{P}(5 \mathrm{c})=60.2$ (s) ppm. 1H NMR: $0.95(\mathrm{dd} ; 12 \mathrm{H} ; \mathrm{J}=15.6 ; 7.2$ $\mathrm{Hz}$; CH3(iPr)); 1.46 (dd; 12H; J = 18.4; 7.2 Hz; CH3(iPr)); 2.31 (dht; 4H; J = 9.6; 7.2; Hz; CH(iPr)); 3.33 (d; 4H; J = $10.4 \mathrm{~Hz} ; \mathrm{CH} 2(\mathrm{Bn})) ; 6.90$ (d; 2H; J = $8.4 \mathrm{~Hz} ; \mathrm{CH}(\mathrm{Ar})$ ); 6.96 (d; 2H; J = $8.4 \mathrm{~Hz}$; $\mathrm{CH}(\mathrm{Ar})) .13 \mathrm{C}\{1 \mathrm{H}\}$ NMR: $\delta$ : $18.0(\mathrm{~s} ; \mathrm{CH} 3) ; 19.2(\mathrm{~s} ; \mathrm{CH} 3) ; 26.0(\mathrm{~d} ; \mathrm{J}=25.3 \mathrm{~Hz} ; \mathrm{CH} 2(\mathrm{Bn})) ; 33.8(\mathrm{~d} ; \mathrm{J}=$ $33.7 \mathrm{~Hz} ; \mathrm{CH}) ; 126.5$ (s; CH); 127.6 (d; J = 22.9 Hz; C); 128.9 (s; CH); 139.8 (s; C); 144.19 (d; J = 16.9 Hz; C); 152.4 (d; J = 3.8 Hz; C). 31P \{1H\}: 87.1 (s). IR: 3094, 3041, 2958, 2921, 2894, 2871, 1455, 1413, 1237, 1151, 1053, 1026, 878, 802. Anal. Calc. for C26H36Br2Cl4P2Pd2 C 33.76 \%, H $3.92 \%$; found C $34.02 \%$, H $4.15 \%$. HRMS: $380.9544[(\mathrm{M} / 2)-\mathrm{Br}]+, 421.9819[(\mathrm{M} / 2)-\mathrm{Br}+(\mathrm{CH} 3 \mathrm{CN})]+$.

\section{Di- $\mu$-bromo-bis $\{2-[($ diphenylphosphano)methyl]-3,6-difluorophenyl-C1,P\}dipalladium(II)}

(9a):

The procedure was analogous to that employed for $8 \mathrm{a}$ but using $2 \mathrm{a}$. The compound was obtained as a yellow solid. The yield was calculated relative to the initial $\mathrm{Pd}(\mathrm{OAc}) 2: 0.480 \mathrm{~g}(69 \%) . \delta \mathrm{P}(2 \mathrm{a})=-11.5$ ppm $(\mathrm{d}, \mathrm{J}=12.1 \mathrm{~Hz}), \delta \mathrm{P}(6 \mathrm{a})=57.9$ (s), 59.3 (s) ppm; 3:2 ratio. $1 \mathrm{H}$ NMR: 3.92 (d; J = $12.0 \mathrm{~Hz} ; 4 \mathrm{H}$; CH2(Bn)); 6.50-6.60 (m; 4H; CH(Ar)); 7.30-7.75 (m; 20H; CH(Ph)). 13C $\{1 \mathrm{H}\}$ NMR: 38.9 (d; J = 36.7 $\mathrm{Hz}$; $\mathrm{CH} 2(\mathrm{Bn})$ ); 112.7 (dd; J = 24.4; 8.3; Hz; CH); 114.6 (dd; J = 32.8; 5.8; Hz; CH); 128.9 (d; J = 10.4 Hz; CH); 129.2 (s; C); 129.7 (s; C); 131.5 (s; C); 131.8 (s; CH); 133.3 (d; J = 11.6 Hz; CH); 154.9 (dd; J = 244.6; 24.4; Hz; CF); 161.7 (d; J = 236.2 Hz; CF). 31P $\{1 \mathrm{H}\}$ NMR: 57.0 (s); 57.9 (s) (ratio 1:3). 19F NMR:-118.5 (m; 1Fo); -94.9 (m; 1Fm). IR: 3087, 3046, 2959, 2916, 1561, 1451, 1433, 1384, 1111, 1099, 969, 864. Anal . Cal c . for C38H28Br2F4P2Pd2 C $45.86 \%$, H 2.84 \%; found C 45.54 \%, H 2.96 \%. HRMS: $416.9836[(\mathrm{M} / 2)-\mathrm{Br}]+$.

\section{Di- $\mu$-bromo-bis $\{2-[($ dicyclohexylphosphano)methyl]-3,6-difluorophenyl-C1,P\}dipalladium(II)}

(9b): The procedure was analogous to that employed for 8 a but using $2 \mathrm{~b}$. The compound was obtained as a yellow solid after column chromatography purification. The yield was calculated relative to the initial $\mathrm{Pd}(\mathrm{OAc}) 2: 0.200 \mathrm{~g}(28 \%) . \delta \mathrm{P}(2 \mathrm{~b})=+2.4 \mathrm{ppm}(\mathrm{d}, \mathrm{J}=9.5 \mathrm{~Hz}), \delta \mathrm{P}(6 \mathrm{~b})=69.3(\mathrm{~s}) .1 \mathrm{H}$ NMR: 0.80 2.50 (m; $44 \mathrm{H} ; \mathrm{CH}(\mathrm{Cy})+\mathrm{CH} 2(\mathrm{Cy})) ; 3.19$ (d; J = $10.2 \mathrm{~Hz} ; 4 \mathrm{H} ; \mathrm{CH} 2(\mathrm{Bn})) ; 6.64(\mathrm{~d} ; \mathrm{J}=5.8 \mathrm{~Hz} ; 2 \mathrm{H}$; $\mathrm{CH}(\mathrm{Ar})) ; 6.66(\mathrm{~d} ; \mathrm{J}=5.8 \mathrm{~Hz} ; 2 \mathrm{H} ; \mathrm{CH}(\mathrm{Ar})) .13 \mathrm{C}\{1 \mathrm{H}\} \mathrm{NMR}: 25.8(\mathrm{~s} ; \mathrm{CH} 2) ; 26.1$ (d; J = 14.1 Hz; CH); 26.6 (d; J = 10.9 Hz; CH2); 28.1 (s; CH2); 28.8 (s; CH2); 29.8 (d; J = 33.5 Hz; CH2); 35.2 (d; J = 25.0 
Hz; CH2(Bn)); 112.0 (dd; J = 9.0; 24.4 Hz; CH); 114.2 (d; J = 7.6; 33.4 Hz; CH); 134.1 (d; J = 14.8 Hz; C); $138.6(\mathrm{~d} ; \mathrm{J}=41.8 \mathrm{~Hz} ; \mathrm{C}) ; 154.3$ (dd; J = 244.6; $24.4 \mathrm{~Hz} ; \mathrm{CF}) ; 161.8(\mathrm{~d} ; \mathrm{J}=235.0 \mathrm{~Hz} ; \mathrm{CF}) .31 \mathrm{P}\{1 \mathrm{H}\}$ NMR: 85.1 (s). 19F NMR:-118.5 (m; 1Fo); -93.9 (bd; J = 19.6 Hz; 1Fm). IR: 3061, 2930, 2852, 1558, 1448, 1218, 977, 855. Anal. Calc. for C38H52Br2F4P2Pd2 C $44.77 \%$, H $5.14 \%$; found C $46.02 \%$; H $5.52 \%$. HRMS: $429.0777[(\mathrm{M} / 2)-\mathrm{Br}]+, 470.1043[(\mathrm{M} / 2)-\mathrm{Br}+(\mathrm{CH} 3 \mathrm{CN})]+$.

\section{Di- $\mu$-bromo-bis $\{2-[($ diisopropylphosphano)methyl]-3,6-difluorophenyl-C1,P $\}$ dipalladium(II) (9c):}

The procedure was analogous to that employed for $8 \mathrm{a}$ but using $2 \mathrm{c}$. The compound was obtained as a yellow solid. The yield was calculated relative to the initial $\mathrm{Pd}(\mathrm{OAc}) 2: 0.120 \mathrm{~g}(20 \%)$. $\delta \mathrm{P}(2 \mathrm{c})=+10.6$ ppm (d, J = 10.5 Hz), $\delta \mathrm{P}(6 \mathrm{c})=78.0$ (s). 1H NMR: 1.01 (dd; J = 15.6; 7.2 Hz; 12H; CH3(iPr)); 1.48 (dd; $\mathrm{J}=18.0 ; 7.2 \mathrm{~Hz} ; 12 \mathrm{H} ; \mathrm{CH} 3(\mathrm{iPr})$ ); 2.35 (dht; J = 9.2; 7.2 Hz; 4H; CH(iPr)); 3.20 (d; J = 10.4 Hz; 4H; $\mathrm{CH} 2(\mathrm{Bn})$ ); 6.65 (d; J = 5.6 Hz; 2H; CH(Ar)); 6.67 (d; J = 5.6 Hz; 2H; CH(Ar)). 13C \{1H\} NMR: 17.9 (s; CH3); 19.1 (s; CH3); 25.9 (d; J = 25.3 Hz; CH2(Bn)); 29.0 (d; J = 32.9 Hz; CH); 112.4 (dd; J = 24.5; 9.1; Hz; CH); 114.4 (dd; J = 32.9; 7.6; Hz; CH); 134.0 (s; C); 134.1 (s; C); 154.7 (d; J = $242.0 \mathrm{~Hz}$; CF);162.0 (d; J = 234.0 Hz; CF). 31P NMR: 94.8 (s). 19F NMR: -118.3 (bd; J = 19.5 Hz; 1Fo); -94.8 (d; J = 17.3 Hz; 1Fm). IR: 3061, 2962, 2933, 2895, 2869, 1451, 1207, 971, 849, 806. Anal. Calc. for C26H36Br2F4P2Pd2 C $36.35 \%$, H $4.22 \%$; found C 37.16 \%, H $4.42 \%$. HRMS: 349.0143 [(M/2) $\mathrm{Br}]+, 390.0409[(\mathrm{M} / 2)-\mathrm{Br}+(\mathrm{CH} 3 \mathrm{CN})]+$.

\section{Di- $\mu$-bromo-bis $\{2-[($ diphenylphosphano)methyl]-4,5,6-trifluorophenyl-C1,P $\}$ dipalladium(II) (10a):}

The procedure was analogous to that employed for $8 \mathrm{a}$ but using $3 \mathrm{a}$. The compound was obtained as a yellow solid. The yield was calculated relative to the initial Pd(OAc)2: $0.195 \mathrm{~g}(27 \%)$. The compound showed low solubility in common organic solvents. $\delta \mathrm{P}(3 \mathrm{a})=-9.9 \mathrm{ppm}(\mathrm{s}), \delta \mathrm{P}(7 \mathrm{a})=46.0(\mathrm{~s}), 46.1 \mathrm{ppm}$; 1:9 ratio. 1H NMR: 3.88 (d; 4H; J = 12.0 Hz; CH2(Bn)); $6.72(\mathrm{~m} ; 2 \mathrm{H} ; \mathrm{J}=7.2 \mathrm{~Hz} ; \mathrm{CH}(\mathrm{Ar})$ ); 7.30-7.80 (m; 20H; $\mathrm{CH}(\mathrm{Ph}))$. 13C $\{1 \mathrm{H}\}$ NMR: 44.1 (d; J = 41.4 Hz; CH2(Bn)); 108.23 (m; CH); 128.9 (m; CH); 131.9 (m; CH); 133.7 (d; J = 11.5 Hz; CH). 31P \{1H\} NMR: 54.3 (s); 54.4 (s) (ratio 1:9). 19F NMR: 163.4 (t; J = 22.5 Hz; 1Fm); -140.1 (m; 1Fp);-109.8 (d; J = 22.5 Hz; 1Fo). IR: 3083, 3056, 2947, 2927, 2854, 1479, 1432, 1416, 1333, 1040, 821, 745. Anal. Calc. for C38H26Br2F6P2Pd2 C 44.26 \%; H 2.54 \%; found C $46.77 \%$, H $3.30 \%$. HRMS: 1031.2119 [M-Br+2CH3CN], 765.0521 [Pd(PC)P]+.

\section{Di- $\mu$-bromo-bis $\{2-[($ dicyclohexylphosphano)methyl]-4,5,6-trifluorophenyl-C1,P $\}$ dipalladium(II)}

(10b): The procedure was analogous to that employed for $8 \mathrm{a}$ but using $3 \mathrm{~b}$. The compound was obtained as a yellow solid. The yield was calculated relative to the initial $\mathrm{Pd}(\mathrm{OAc}) 2: 0.480 \mathrm{~g}(65 \%)$. The compound showed low solubility in common organic solvents. $\delta \mathrm{P}(3 \mathrm{~b})=+2.1 \mathrm{ppm}(\mathrm{s}), \delta \mathrm{P}(7 \mathrm{~b})=65.2(\mathrm{~s})$. 1H NMR: 1.00-2.45 (m; 44 H; CH(Cy) + CH2(Cy)); 3.19 (d; 4H; J = 10.8 Hz; CH2(Bn)); 6.68 (d; 2H; J $=6.8 \mathrm{~Hz} ; \mathrm{CH}(\mathrm{Ar})) ; 6.69$ (d; 2H; J = 6.8 Hz; CH(Ar)). 13C $\{1 \mathrm{H}\} \mathrm{NMR}: 25.8$ (s; CH2); 26.1 (d; J = 14.8 $\mathrm{Hz}$; CH); 26.6 (d; J = 11.6 Hz; CH2); 28.2 (s; CH2); 28.8 (s; CH2); 29.8 (s; CH2); 35.1 (d; J = 25.7 Hz; 
626

627

628

629

630

631

632

633

634

635

636

637

638

639

640

641

642

643

644

645

646

647

648

649

650

651

652

653

654

655

656

657

658

CH2(Bn)). 31P $\{1 \mathrm{H}\}$ NMR: 82.1 (s). 19F NMR: -163.9 (ddd; J = 23.7; 19.9; $5.3 \mathrm{~Hz} 1 \mathrm{Fm}$ ); -140.7 (m; J $=10.5 \mathrm{~Hz} ; 1 \mathrm{Fp}$ ); -108.9 (dd; J = 26.7; 6.3; Hz; 1Fo). IR: 3040, 2923, 2850, 2797 v; 1476; 1412; 1333; 1040; 825. Anal. Calc. for C38H50Br2F6P2Pd2 C $43.25 \%$, H $4.78 \%$; found C $43.52 \%$, H $4.83 \%$. HRMS: $447.0684[(\mathrm{M} / 2)-\mathrm{Br}]+, 488.0951[(\mathrm{M} / 2)-\mathrm{Br}+(\mathrm{CH} 3 \mathrm{CN})]+$.

\section{Di- $\mu$-bromo-bis $\{2-[($ diisopropylphosphano)methyl]-4,5,6-trifluorophenyl-C1,P $\}$ dipalladium(II)}

(10c): The procedure was analogous to that employed for $8 \mathrm{a}$ but using $3 \mathrm{c}$. The compound was obtained as a yellow solid. The yield was calculated relative to the initial $\mathrm{Pd}(\mathrm{OAc}) 2: 0.215 \mathrm{~g}(34 \%)$. The compound showed low solubility in common organic solvents. $\delta \mathrm{P}(3 \mathrm{c})=+10.6 \mathrm{ppm}(\mathrm{s}), \delta \mathrm{P}(7 \mathrm{c})=74.4$ (s). 1H NMR: 1.00 (dd; 12H; J = 15.2; 6.8 Hz; CH3(iPr)); 1.47 (dd; 12H; J = 18.0; 6.8 Hz; CH3(iPr)); 2.34 (dht; 4H; J = 7.2 Hz; CH(iPr)); 3.20 (d; 4H; J = $11.2 \mathrm{~Hz} ; \mathrm{CH} 2(\mathrm{Bn})$ ); 6.72 (pt; 2H; J = $8.0 \mathrm{~Hz}$; CH(Ar)). 13C \{1H\} NMR: 18.0 (s; CH3); 19.1 (s; CH3); 25.8 (d; J = 20.1 Hz; CH2(Bn)); 29.7 (d; J = $10.1 \mathrm{~Hz} ; \mathrm{CH}) .31 \mathrm{P}\{1 \mathrm{H}\}$ NMR: 91.0 (s). 19F NMR: -163.8 (ddd; J = 22.5; 7.5; $3.8 \mathrm{~Hz} ; 1 \mathrm{Fm}) ;-140.6$ (dpt; J = 18.0; 8.6 Hz; 1Fp); -109.9 (dd; J = 26.3; 6.4 Hz; 1Fo). IR: 3086, 3038, 2955, 2927, 2865, 1474, 1458, 1410, 1330, 1040, 831, 819. Anal. Calc. for C26H34Br2F6P2Pd2: C $34.89 \%$, H 3.83 \%; found C $35.37 \%$, H $4.01 \%$. HRMS: 367.0060 [(M/2) - Br]+, 408.0323 [(M/2) - $\mathrm{Br}+(\mathrm{CH} 3 \mathrm{CN})]+$.

Di- $\mu$-bromo-bis $\{2-[($ diphenylphosphano)methyl]phenyl-C1,P\}-dipalladium(II) (12a): The procedure was analogous to that employed for $8 \mathrm{a}$ but using $4 \mathrm{a}$. The compound was obtained as a brown solid. The yield was calculated relative to the initial $\mathrm{Pd}(\mathrm{OAc}) 2: 0.355 \mathrm{~g}(55 \%) . \delta \mathrm{P}(4 \mathrm{a})=-10.0 \mathrm{ppm}(\mathrm{s}), \delta \mathrm{P}(11 \mathrm{a})=$ 51.2 (s). 1H NMR: 3.86 (d; 4H; J = 12.0 Hz; CH2(Bn)); 6.82-7.10 (m, 7H); 7.32-7.45 (m, 12H), 7.69$7.83(\mathrm{~m}, 9 \mathrm{H}) .13 \mathrm{C}\{1 \mathrm{H}\}$ NMR: $43.4(\mathrm{~d} ; \mathrm{J}=36.9 \mathrm{~Hz} ; 2 \mathrm{CH} 2(\mathrm{Bn})) ; 124.0-143.9(\mathrm{C}(\mathrm{Ar}), \mathrm{CH}(\mathrm{Ar}))$. 31P $\{1 \mathrm{H}\}$ NMR: 55.9 (s), 56.4 (s), (ratio 2:3). IR: 3048, 1570, 1434, 1101, 1018, 737, 584. Anal. Calc. for C38H32Br2P2Pd2: C $49.44 \%$, H $3.49 \%$; found C $48.56 \%$, H $3.73 \%$. HRMS: 381.0025 [(M/2) $\mathrm{Br}]+, 422.0289[(\mathrm{M} / 2)-\mathrm{Br}+(\mathrm{CH} 3 \mathrm{CN})]+$.

\section{Di- $\mu$-bromo-bis $\{2-[($ diisopropylphosphano)methyl]phenyl-C1,P $\}$-dipalladium(II) $\quad$ (12c): $\quad$ The} procedure was analogous to that employed for $8 \mathrm{a}$ but using 4c. The compound was obtained as a pale brown solid. The yield was calculated relative to the initial $\mathrm{Pd}(\mathrm{OAc}) 2: 0.103 \mathrm{~g}(19 \%) . \delta \mathrm{P}(4 \mathrm{c})=9.9 \mathrm{ppm}$ $(\mathrm{s}), \delta \mathrm{P}(11 \mathrm{c})=76.0(\mathrm{~s}) .1 \mathrm{H}$ NMR: 1.05-1.12 (m; 12H; CH3(iPr)); 1.43-1.51 (m; 12H; CH3(iPr)); $2.17-$ 2.41 (m; 4H; $\mathrm{CH}(\mathrm{iPr})$ ); 3.11-3.19 (m; 4H; CH2(Bn)); 6.87-7.057 (m; 6H; H(Ar)); 7.46-7.95 (m; 2H; H(Ar)). 13C $\{1 \mathrm{H}\}$ NMR: 18.0 (s; CH3(iPr)); 19.3 (s; CH3(iPr)); 25.6 (d; J = 25.1 Hz; CH(iPr)); 32.6 (d; $\mathrm{J}=32.2 \mathrm{~Hz} ; 2 \mathrm{CH} 2(\mathrm{Bn})$ ); 123.7-152.2 (C, CH Ar). 31P $\{1 \mathrm{H}\}$ NMR: 89.7 (s), 91.0 (s), (ratio 1:2). IR: 3058, 2954, 2924, 2865, 1570, 1443, 1385, 1247, 1015, 700, 662, 643. Anal. Calc. for C26H40Br2P2Pd2: C 39.67 \%, H $5.12 \%$; found C $38.97 \%$, H $5.28 \%$. HRMS: 313.0337 [(M/2) - Br]+, $354.0604[(\mathrm{M} / 2)-\mathrm{Br}+(\mathrm{CH} 3 \mathrm{CN})]+$. 
trans-Bromo\{2-[(diisopropylphosphano)methyl]-3,6-difluorophenyl-

C1,P\}(tricyclohexylphosphano)palladium(II) (13c): A solution of 9c (0.150 g; $0.17 \mathrm{mmol})$ and PCy3 $(0.140 \mathrm{~g}, 0.5 \mathrm{mmol})$ in $10 \mathrm{~mL}$ of toluene was stirred at room temperature for $1 \mathrm{~h}$. After evaporation, the residue was extracted with $10 \mathrm{~mL}$ of dichloromethane and dry ethanol was added. After several hours in the freezer a yellow solid of 13c was filtered off. Yield: $0.156 \mathrm{~g}(63 \%)$. 1H NMR: 0.80-2.50 (m; 33H; $\mathrm{CH}(\mathrm{Cy})+\mathrm{CH} 2(\mathrm{Cy})) ; 0.92(\mathrm{dd} ; 6 \mathrm{H} ; \mathrm{J}=13.8 ; 7.1 \mathrm{~Hz} ; \mathrm{CH} 3(\mathrm{iPr})) ; 1.36(\mathrm{dd} ; 6 \mathrm{H} ; \mathrm{J}=11.1 ; 26.9 \mathrm{~Hz}$; CH3(iPr)); 2.25-2.55 (m; CH(iPr)); 3.20 (d; J = 9.5 Hz; CH2(Bn)); 6.62 (m; CH(Ar)). 31P $\{1 \mathrm{H}\}$ NMR (101.1 MHz; C3D6O), $\delta(\mathrm{ppm}): 23.7(\mathrm{dd} ; \mathrm{JPP}=414.0 ; \mathrm{JPF}=27.3 \mathrm{~Hz}) ; 77.5(\mathrm{~d} ; \mathrm{JPP}=414.0 \mathrm{~Hz}) . \mathrm{IR}$ : 3064, 2924, 2846, 1445, 1387, 1239, 1204, 1178, 849, 806. Anal. Calc. for C31H51BrF2P2Pd: C 52.44 \%, H $7.24 \%$; found C $53.43 \%$, H $8.08 \%$. HRMS: 629.7009 [M-Br]+, 669.8999 [M-Br+(CH3CN)]+.

Preparation of Ionic complexes 14-18: A solution of the suitable palladium complex (0.12 mmol) in $\mathrm{CH} 2 \mathrm{Cl} 2(5 \mathrm{~mL})$ was Added, whilst stirring, to a $\mathrm{CH} 2 \mathrm{Cl} 2(5 \mathrm{~mL})$ suspension of norbornadiene $(0.36$ mmol) and AgBF4 (0.36 mmol). A precipitate appears immediately. The suspension was stirred for $1 \mathrm{~h}$ in the dark, after which time the solid was filtered through a celite pad. The resulting solution was concentrated under reduced pressure and pentane was added under strong stirring to prevent the formation of resin. The yellow ionic complex was isolated by filtration and dried under vacuum.

\section{[\{2-[(Diisopropylphosphano)methyl]-3,6-dichlorophenyl-C1,P\}-(1,2,4,5-ๆ4)-2,5-}

bicyclo[2.2.1]heptadiene]palladium(II) Tetrafluoroborate (14c): The compound was obtained as a yellow solid. Yield: 0.045 g (34 \%). 1H NMR: 1.06 (dd; 6H; J = 16.4; 6.4 Hz; CH3(iPr)); 1.40 (dd; 6H; $\mathrm{J}=18.4 ; 7.2 \mathrm{~Hz}$; CH3(iPr)); 2.21 (bs; 2H; CH2(nbd)); 2.44 (bs; 2H; CH(iPr)); 3.41 (bs; 2H; CH2(Bn)); 4.10 (bs; 2H; $\mathrm{CH}(\mathrm{nbd})$ ); 6.98 (d; 1H; J = $8.4 \mathrm{~Hz}$; $\mathrm{CH}(\mathrm{Ar})$ ); 7.1 (d; 1H; J = 8.4 Hz; CH(Ar)); 7.60-7.80 $(\mathrm{m} ; 4 \mathrm{H} ; \mathrm{CH}=\mathrm{CHcis}(\mathrm{nbd})+\mathrm{CH}=\mathrm{CHtrans}(\mathrm{nbd})) .13 \mathrm{C}\{1 \mathrm{H}\}$ NMR: 17.8 (s; CH3); 19.4 (bs; CH3); 24.9 (d; $\mathrm{J}=22.7 \mathrm{~Hz}$; CH2(Bn)); 32.9 (bs; CH); 129.4 (bs; CH). 31P\{1H\} NMR: 86.7 (s). IR: 3113, 3084, 3040, 2962, 2927, 2872, 1155, 1117, 1088, 1047, 1032. Anal. Calc. for C20H26BCl2F4PPd C 42.78 \%, H $4.67 \%$; found C $40.26 \%$, H $4.80 \%$. HRMS: 382.9549 [M-(nbd)]+, $423.9816[\mathrm{M}-(\mathrm{nbd})+(\mathrm{CH} 3 \mathrm{CN})]+$.

\section{[\{2-[(Diisopropylphosphano)methyl]-3,6-difluorophenyl-C1,P\}(1,2,4,5-१4)-2,5-}

bicyclo[2.2.1]heptadiene]palladium(II) Tetrafluoroborate (15c): The compound was obtained as a yellow solid. Yield: 0.118 g (93 \%). 1H NMR: 1.18 (dd; 6H; J = 16.4; 6.8 Hz; CH3(iPr)); 1.35 (dd; 6H; $\mathrm{J}=18.4 ; 7.2 ; \mathrm{Hz} ; \mathrm{CH} 3(\mathrm{iPr})) ; 2.26$ (d; 1H; J = 8.8 Hz; CHH(nbd)); 2.33 (d; 1H; J = 9.2 Hz; CHH(nbd)); 2.62 (ht; 2H; J = 8.8 Hz; CH(iPr)); 3.31 (d; 2H; J = 10.8 Hz; CH2(Bn)); 4.38 (s; 2H; CH(nbd)); 6.73 (m; 1H; $\mathrm{CH}(\mathrm{Ar})) ; 6.85$ (m;1H; $\mathrm{CH}(\mathrm{Ar})) ; 7.11$ (s;2H; $\mathrm{CH}=\mathrm{CHcis}(\mathrm{nbd})$ ); 7.57 (s; 2H; $\mathrm{CH}=\mathrm{CHtrans}(\mathrm{nbd})$ ). 13C $\{1 \mathrm{H}\}$ NMR: 18.1 (d; J = 1.91 Hz; CH3); 19.5 (s; CH3); 25.9 (d; J = 23.2 Hz; CH2(Bn)); 28.2 (d; J = $34.1 \mathrm{~Hz} ; \mathrm{CH}) ; 54.1$ (s; CH2); 78.6 (s; CH); 115.1 (dd; J = 33.4; 7.3 Hz; CH=CHcis(nbd)); 115.6 (dd; J = 24.4; 9.9 Hz; $\mathrm{CH}=\mathrm{CHcis}(\mathrm{nbd})$ ); 116.1 (s; CH); 121.5 (dd; J = 10.3; 7.7 Hz; CH); 135.4 (d; J = $12.9 \mathrm{~Hz}$; 
$\mathrm{CH}=\mathrm{CH}$ trans(nbd)); 140.5 (d; J = 36.6 Hz; CH=CHtrans(nbd)); 143.5 (s; C); 154.6 (dd; J = 246.6; 23.7 Hz; CF); 161.6 (d; J = 233.0 Hz; CF). 31P \{1H\} NMR: 92.5 (s). 19F NMR: -152.7 (s; 4F; BF4); -114.6 (bd; J = 18.8 Hz; 1Fo); -108.1 (d; J = 16.2 Hz; 1Fm). IR: 3110, 3079, 2968, 2931, 2869, 1565, 1450, 1314, 1225, 1209, 1086, 1058, 1039, 965, 854, 820, 767, 749, 724. Anal. Calc. for C20H26BF6PPd C $45.44 \%, \mathrm{H} 4.96 \%$; found C $45.90 \%$, H $5.40 \%$. HRMS: 349.0144 [M-(nbd)]+, 390.0406 [M$(\mathrm{nbd})+(\mathrm{CH} 3 \mathrm{CN})]+$.

\section{[\{2-[(Diisopropylphosphano)methyl]phenyl-C1,P\}(1,2,4,5-ワ4)-2,5-}

bicyclo[2.2.1]heptadiene]palladium(II) Tetrafluoroborate (16c): The compound was obtained as a yellow solid. Yield: $0.065 \mathrm{~g}$ (54 \%). The NMR were recorded in [D6]acetone. 1H NMR: 1.14 (dd; 6H; J = 15.6; 6.8 Hz; CH3(iPr)); 1.28 (br, CH2(nbd)) 1.40 (dd; 6H; J = 18.0; 7.2; Hz; CH3(iPr)); 2.35-2.48 (m; 2H; $\mathrm{CH}(\mathrm{iPr})) ; 3.36$ (d; 2H; J = 10.8 Hz; CH2(Bn)); 6.86-7.80 (m, H(Ar)). 13C $\{1 \mathrm{H}\}$ NMR: 17.8 (br; CH3(iPr)); 19.1 (d; J = 2.9 Hz, CH3(iPr)); 25.2 (d; J = 27.2 Hz; CH(iPr)); 31.3 (d; J = 34.6 Hz; CH2(Bn)); 125.2-149.4 (m, C, CH(Ar)). 31P\{1H\} NMR: 95.4 (s). 19F NMR: -151.8 (s; 4F; BF4). IR: 2961, 2931, 1524, 1447, 1349, 1017 (v(BF4)), 884, 812, 745, 647. HRMS: 313.0332 [M-(nbd)]+.

\section{[\{2-[(Diphenylphosphano)methyl]phenyl-C1,P $\}(1,2,4,5-\eta 4)-2,5-$}

bicyclo[2.2.1] heptadiene]palladium(II) Tetrafluoroborate (17a): The compound was obtained as a yellow solid after recrystallization in $\mathrm{CH} 2 \mathrm{Cl} 2 / \mathrm{Et} 2 \mathrm{O}$. Yield: $0.044 \mathrm{~g}(33 \%)$. The NMR were recorded in [D6]acetone. 1H NMR: 1.91 (s; 2H; CH2(nbd)); 3.56 (s; 2H; 2CH(nbd)); 4.22 (d; 2H; J = $13.2 \mathrm{~Hz}$; $\mathrm{CH} 2(\mathrm{Bn})) ; 6.74$ (s; 3H; $\mathrm{CH}=\mathrm{CHcis}(\mathrm{nbd})+1 \mathrm{H}(\mathrm{Ar})) ; 6.92 \quad(\mathrm{br}, 1 \mathrm{H}(\mathrm{Ar})), \quad 7.06-7.09$ (m; 2H; $\mathrm{CH}=\mathrm{CH}$ trans(nbd)); 7.20 (d, J = 9.6, 1H(Ar)); 7.57-7.61 (m, 4H(Ar)); 7.65-7.69 (m, 3H(Ar)); 7.84-7.89 (m, 4H(Ar)). 13C $\{1 \mathrm{H}\}$ NMR: 40.6 (d; J = 39.1 Hz; CH2(Bn)); 50.9 (s; 2CH(nbd)); 75.7 (s; CH2(nbd)); 126.1-146.5 (m, C, CH) 31P\{1H\} NMR: 56.3 (s). 19F NMR: -151.6 (s; 4F; BF4). IR: 3072, 2956, 1436, 1034 (v(BF4)), 742, 693. HRMS: 381.0019 [M-(nbd)]+.

\section{[\{2-[(Diphenylphosphano)methyl]-4,5,6-trifluorophenyl-C1,P $\}-(1,2,4,5-\eta 4)-2,5-$}

bicyclo[2.2.1] heptadiene]palladium(II) Tetrafluoroborate (18a): The compound was obtained as a light yellow solid. Yield: 0.136 g (92 \%). 1H NMR: 2.09 (m; 1H; $\mathrm{CHH}^{\prime}(\mathrm{nbd})$ ); 2.20 (d; 1H; J = $9.2 \mathrm{~Hz}$; $\mathrm{CH} 2(\mathrm{nbd})$ ); 4.15 (d; 2H; J = 13.6 Hz; CH2(Bn)); 4.41 (s; 2H; CH(nbd)); 6.30 (s; 2H; $\mathrm{CH}=\mathrm{CHcis}(\mathrm{nbd})$ ); 6.83 (m; 1H; $\mathrm{CH}(\mathrm{Ar})$ ); 7.41 (broad; 2H; $\mathrm{CH}=\mathrm{CH} \operatorname{trans}(\mathrm{nbd})) ; 7.50-7.70(\mathrm{~m} ; 10 \mathrm{H} ; \mathrm{CH}(\mathrm{Ph})) .13 \mathrm{C}\{1 \mathrm{H}\}$ NMR: 41.2 (d; J = 35.2 Hz; CH2(Bn)); 54.2 (s; CH2); 76.9 (s; CH); 110.4 (m; CH=CHcis(nbd)); 115.9 (m; $\mathrm{CH}=\mathrm{CHcis}(\mathrm{nbd})$ ); 120.5 (pt; J = 9.1 Hz; CH); 126.2 (d; J = 53.7 Hz; C); 129.0 (m; CH); 130.2 (d; J $=11.6 \mathrm{~Hz} ; \mathrm{CH}=\mathrm{CH} \operatorname{trans}(\mathrm{nbd})) ; 133.1$ (d; J = 11.6 Hz; CH=CHtrans(nbd)); 133.4 (d; J = 3.0 Hz; CH); 139.7 (m; C). 31P $\{1 \mathrm{H}\}$ NMR: 57.3 (s). 19F NMR: -161.9 (ddd; J = 36.5; 18.8; 6.7 Hz; 1F); -152.7 (s; 4F; BF4); -134.6 (dt; J = 9.6 Hz; 1F);-122.3 (dd; J = 26.8; 7.9 Hz; 1F). IR: 3057, 2961, 2924, 2856 v; 1475; 1435; 1333; 1101; 1036; 823; 746; 696; 517. Anal. Calc. for C26H21BF7PPd C 50.81 \%, H 3.44 
738

739

740

741

742

743

744

745

746

747

748

749

750

751

752

753

754

755

756

757

758

759

760

761

762

763

764

765

766

767

768

\%; found C 50.11 \%, H $3.82 \%$. HRMS: 527.4001; [M]+ 435.0001, [M-(nbd)]+, 476.4012 [M$(\mathrm{nbd})+(\mathrm{CH} 3 \mathrm{CN})]+$.

\section{[\{2-[(Dicyclohexylphosphano)methyl]-4,5,6-trifluorophenyl-C1,P $\}(1,2,4,5-\eta 4)-2,5-$}

bicyclo[2.2.1]heptadiene]palladium(II) Tetrafluoroborate (18b): The compound was obtained as a light yellow solid. Yield: 0.138 g (92\%). 1H NMR: 1.10-2.00 (m; 22H; CH(Cy)+CH2(Cy)); 2.21 (m; 2H; CH2(nbd)); 3.35 (d; 2H; J = 11.4 Hz; CH2(Bn)); 4.33 (s; CH(nbd)); 6.83 (m; CH(Ar)); 6.95 (m; 2H; $\mathrm{CH}=\mathrm{CHcis}(\mathrm{nbd})) ; 7.46(\mathrm{~m} ; 2 \mathrm{H} ; \mathrm{CH}=\mathrm{CH} \operatorname{trans}(\mathrm{nbd})) .13 \mathrm{C}\{1 \mathrm{H}\} \quad \mathrm{NMR}: 25.7$ (s; CH2); 26.4 (m; $\mathrm{CH}+\mathrm{CH} 2) ; 28.7$ (d; J = 3.1 Hz; CH2); 30.1 (s; CH2); 33.6 (d; J = 35.2 Hz; CH2(Bn)); 35.2 (d; J = 22.9 Hz; CH2); 54.3 (s; CH2); 78.2 (s; CH); 109.2 (t; J = 19.1 Hz; CH); 115.2 (s; CH=CHcis(nbd)); 120.6 (pt; J = 9.1 Hz; CH=CHcis(nbd)); 143.6 (s; C). 31P $\{1 \mathrm{H}\}$ NMR: 81.3 (s). 19F NMR: -162.6 (ddd; J = 26.7; 26.3; 7.9 Hz; 1F);-151.9 (s; BF4); -134.8 (dt; J = 26.3; $13.2 \mathrm{~Hz} ; 1 \mathrm{~F}) ;-123.8$ (dd; J = 26.7; 6.3 Hz; 1F). IR: 3101, 2927, 2852 v; 1483; 1332; 1088; 1035; 826. Anal. Calc. for C26H33BF7PPd C 49.83 \%, H $5.31 \%$; found C $49.80 \%$, H $5.92 \%$. HRMS: 447.0575 [M-(nbd)]+; 488.0539 [M+(CH3CN)]+.

\section{[\{2-[(Diisopropylphosphano)methyl]-4,5,6-trifluorophenyl-C1,P\}(1,2,4,5-ฤ4)-2,5-}

bicyclo[2.2.1]heptadiene]palladium(II) Tetrafluoroborate (18c): The compound was obtained as a light yellow solid. Yield: 0.077 g (59 \%). 1H NMR: 1.16 (dd; 6H; J = 16.8; 6.8; Hz; CH3(iPr)); 1.32 (dd; $6 \mathrm{H} ; \mathrm{J}=18.0 ; 7.2 ; \mathrm{Hz} ; \mathrm{CH} 3(\mathrm{iPr})$ ); 2.24-2.33 (m; 2H; CH2(nbd)); 2.57 (dht; 2H; J = 9.2; $7.2 \mathrm{~Hz}$; $\mathrm{CH}(\mathrm{iPr})$ ); 3.39 (d; 2H; J = $11.6 \mathrm{~Hz} ; \mathrm{CH} 2(\mathrm{Bn})) ; 4.39$ (s; 2H; $\mathrm{CH}(\mathrm{nbd})) ; 6.92$ (pt; 1H; J = $7.6 \mathrm{~Hz}$; $\mathrm{CH}(\mathrm{Ar})) ; 7.06$ (m; 2H; $\mathrm{CH}=\mathrm{CHcis}(\mathrm{nbd})) ; 7.55$ (m; 2H; $\mathrm{CH}=\mathrm{CH} \operatorname{trans}(\mathrm{nbd})) .13 \mathrm{C}\{1 \mathrm{H}\} \mathrm{NMR}: 18.1$ (d; J = $1.91 \mathrm{~Hz}$; CH3); 19.5 (s; CH3); 41.2 (d; J = 37.5 Hz; CH2(Bn)); 28.2 (d; J = 34.1 Hz; CH); 54.1 (s; CH2); 78.6 (s; CH); 115.9 (s; CH=CHcis(nbd)); 120.5 (pt; J = 9.1 Hz; $\mathrm{CH}=\mathrm{CH} \operatorname{cis}(\mathrm{nbd})$ ); 116.1 (s; CH); 121.5 (dd; J = 10.3; 7.7 Hz; CH); 130.1 (d; J = 11.5 Hz; CH= CHtrans(nbd)); 133.0 (d; J = $11.0 \mathrm{~Hz}$; $\mathrm{CH}=\mathrm{CH} \operatorname{trans}(\mathrm{nbd})$ ); 143.5 (s; C); 154.6 (dd; J = 246.6; $23.7 \mathrm{~Hz} ; \mathrm{CF}) ; 161.6$ (d; J = 233.0 Hz; CF). 31P $\{1 \mathrm{H}\}$ NMR: 88.7 (s). 19F NMR: -162.2 (ddd; J = 26.8; 9.4; 6.7; Hz; 1F); -152.5 (s; BF4); -134.5 (dt; J = 19.9; 9.4 Hz; 1F); -123.7 (dd; J = 26.7; 9.4 Hz; 1F). IR: 3102, 3047, 2969, 2936, 2875, 1486, $1416,1394,1333,1314,1087,1068,1040$. HRMS: 367.0066 [M-(nbd)]+, 408.0324 [M$(\mathrm{nbd})+(\mathrm{CH} 3 \mathrm{CN})]+$.

Procedures of the Catalytic Runs: Two parallel catalytic runs were always performed for each precursor. A Schlenk flask was charged with cesium carbonate $(3.58 \mathrm{~g}, 11 \mathrm{mmol})$ and DMF $(15 \mathrm{~mL})$ was added. To this suspension bromobenzene $(1.05 \mathrm{~mL}, 10 \mathrm{mmol})$ and butyl acrylate $(2.15 \mathrm{~mL}, 15$ mmol) were subsequently added and the mixture was warmed up to $130{ }^{\circ} \mathrm{C}$ for $15 \mathrm{~min}$. Then the catalytic palladium precursor $(0.01 \mathrm{mmol}$ of mononuclear or $0.005 \mathrm{mmol}$ of dinuclear complexes), previously dissolved in $5 \mathrm{~mL}$ of DMF was rapidly added and an aliquot of approximately $0.1 \mathrm{~mL}(\mathrm{t}=0)$ 
769 was taken. Five more aliquots were taken. Each aliquot was passed by a short pad of silica eluting with 770 dichloromethane and injected in the GC to evaluate the conversion 


\section{ACKNOWLEDGEMENTS}

773

774 We thank the Ministerio de Economía y Competitividad (grant number CTQ2015-65040-P) for financial 775 support of this work.

776 


\section{REFERENCES}

[1] a) Metal-Catalyzed Cross-Coupling Reactions and More (Eds.: A. de Meijere, S. Bräse, M. Oestreich), Wiley-VCH, Weinhem, 2014; b) New Trends in Cross-Coupling. Theory and Applications (Ed.: T. J. Colacot), RSC Catalysis Series, The Royal Society of Chemistry, Cambridge, 2015.

[2] a) T. Mizoroki, K. Mori, A. Ozaki, Bull. Chem. Soc. Jpn. 1971, 44, 581-581; b) R. F. Heck, Acc. Chem. Res. 1979, 12, 146-151.

[3] a) G. T. Crisp, Chem. Soc. Rev. 1998, 27, 427-436; b) I. P. Beletskaya, A. V. Cheprakov, Chem. Rev. 2000, 100, 3009-3066; c) The Mizoroki-Heck Reaction (Ed.: M. Oestreich), Wiley, Chichester, 2009; d) C. Amatore, A. Jutand, Acc. Chem. Res. 2000, 33, 314-321; e) A. M. Trzeciak, J. J. Ziółkowski, Coord. Chem. Rev. 2005, 249, 2308-2322; f) F. Alonso, I. P. Beletskaya, M. Yus, Tetrahedron 2005, 61, 11771-11835; g) N. T. S. Phan, M. Van Der Sluys, C. W. Jones, Adv. Synth. Catal. 2006, 348, 609-679; h) J. P. Knowles, A. Whiting, Org. Biomol. Chem. 2007, 5, 31-44; i) S. Jagtap, Catalysts 2017, 7, 267.

[4] a) W. A. Herrmann, C. Brossmer, C.-P. Reisinger, T. H. Riermeier, K. Öfele, M. Beller, Chem. Eur. J. 1997, 3, 1357-1364; b) R. F. Heck, J. P. Nolley, J. Org. Chem. 1972, 37, 2320-2322.

[5] W. A. Herrmann, V. P. W. Böhm, C.-P. Reisinger, J. Organomet. Chem. 1999, 576, 23-41.

[6] P. P. Mpungose, P. Z. Vundla, E. M. G. Maguire, B. H. Friedrich, Molecules 2018, 23, 1676.

[7] W. A. Herrmann, C. Brossmer, K. Öfele, C.-P. Reisinger, T. Priermeier, M. Beller, H. Fischer, Angew. Chem. Int. Ed. Engl. 1995, 34, 1844-1848; Angew. Chem. 1995, 107, 1989.

[8] a) I. P. Beletskaya, A. V. Cheprakov, J. Organomet. Chem. 2004, 689, 4055-4082; b) Y. Ding, M. Chiang, S. A. Pullarkat, Y. Li, P.-H. Leung, Organometallics 2009, 28, 4358-4370; c) D.-L. Mo, T.-K. Zhang, G.-C. Ge, X.-J. Huang, C.-H. Ding, L.-X. Dai, X.-L. Hou, Synlett 2014, 25, $2686-2702$.

[9] S. Gibson, D. F. Foster, G. R. Eastham, R. P. Tooze, D. J. Cole-Hamilton, Chem. Commun. 2001, 779-780.

[10] B. L. Shaw, M. M. Truelock, J. Organomet. Chem. 1975, 102, 517-525.

[11] S. Hietkamp, D. J. Stufkens, K. Vrieze, J. Organomet. Chem. 1979, 168, 351-361. 
[12] K. Hiraki, Y. Fuchita, T. Uchiyama, Inorg. Chim. Acta 1983, 69, 187-190. Eur. J. Inorg. Chem. 0000, 0-0 www.eurjic.org 15 @ 2020 Wiley-VCH Verlag GmbH \& Co. KGaA, Weinheim

[13] J. K.-P. Ng, S. Chen, Y. Li, G.-K. Tan, L.-L. Koh, P.-H. Leung, Inorg. Chem. 2007, 46, 51005109 .

[14] D. Gatineau, L. Giordano, G. Buono, J. Am. Chem. Soc. 2011, 133, 10728-10731.

[15] a) H. Lebel, S. Morin, V. Paquet, Org. Lett. 2003, 5, 2347-2349; b) M. T. Honaker, B. J. Sandefur, J. L. Hargett, A. L. McDaniel, R. N. Salvatore, Tetrahedron Lett. 2003, 44, 83738377.

[16] R. B. Bedford, S. L. Hazelwood, P. N. Horton, M. B. Hursthouse, Dalton Trans. 2003, 41644174 .

[17] S. Freitag, J. Henning, H. Schubert, L. Wesemann, Angew. Chem. Int. Ed. 2013, 52, 56405643; Angew. Chem. 2013, 125, 5750.

[18] W.-C. Shih, O. V. Ozerov, Organometallics 2015, 34, 4591-4597.

[19] a) P. Wyatt, H. Eley, J. Charmant, B. J. Daniel, A. Kantacha, Eur. J. Org. Chem. 2003, 2003, 4216-4226; b) A. J. Rucklidge, G. E. Morris, A. M. Z. Slawin, D. J. Cole-Hamilton, Helv. Chim. Acta 2006, 89, 1783-1800; c) G. B. Consiglio, P. Queval, A. Harrison-Marchand, A. Mordini, J. Lohier, Delacrosix, A. Gaumont, H. Gerard, J. Maddaluno, H. Oulyadi, J. Am. Chem. Soc. 2011, 133, 6472-6480; d) A. K. Ghosh, D. R. Nicponski, J. Kass, Tetrahedron Lett. 2012, 53, 3699-3702; e) K. Izod, C. M. Dixon, E. McMeekin, L. Rodgers, R. W. Harrington, U. Baisch, Organometallics 2014, 33, 378-386; f) K. M. Crawford, T. R. Ramseyer, C. J. A. Daley, T. B. Clark, Angew. Chem. Int. Ed. 2014, 53, 7589-7593; Angew. Chem. 2014, 126, 7719; g) C. Li, Q.-L. Bian, S. Xu, W.-L. Duan, Org. Chem. Front. 2014, 1, 541-545; h) D. Gatineau, D. H. Nguyen, D. Hérault, N. Vanthuyne, J. Leclaire, L. Giordano, G. Buono, J. Org. Chem. 2015, $80,4132-4141$.

[20] a) J. K.-P. Ng, Y. Li, G.-K. Tan, L.-L. Koh, J. J. Vittal, P.-H. Leung, Inorg. Chem. 2005, 44, 9874-9886; b) J. K.-P. Ng, S. Chen, G.-K. Tan, P.-H. Leung, Eur. J. Inorg. Chem. 2007, 2007, 3124-3134.

[21] P. E. Garrou, Chem. Rev. 1981, 81, 229-266.

[22] R. B. Bedford, S. L. Hazelwood, M. E. Limmert, D. A. Albisson, S. M. Draper, P. N. Scully, S. J. Coles, M. B. Hursthouse, Chem. Eur. J. 2003, 9, 3216-3227. 
[23] V. A. Stepanova, L. M. Egan, L. Stahl, I. P. Smoliakova, J. Organomet. Chem. 2011, 696, 31623168 .

838

[24] a) N. C. Baenziger, J. R. Doyle, C. Carpenter, Acta Crystallogr. 1961, 14, 303-308; b) N. C. Baenziger, G. F. Richards, J. R. Doyle, Acta Crystallogr. 1965, 18, 924-926.

[25] N.-H. Kim, K. Ha, Acta Crystallogr., Sect. E 2009, 65, m727.

[26] a) H. Suzuki, K. Itoh, Y. Ishii, K. Simon, J. A. Ibers, J. Am. C em. Soc. 1976, 98, 8494-8500; b) A. S. K. Hashmi, J. W. Bats, F. Naumann, B. Berger, Eur. J. Inorg. Chem. 1998, 1987-1990; c) A. S. K. Hashmi, F. Naumann, M. Bolte, Organometallics 1998, 17, 2385-2387.

[28] J. G. de Vries, Dalton Trans. 2006, 421-429.

[29] S. G. Fiddy, J. Evans, M. A. Newton, T. Neisius, R. P. Tooze, R. Oldman, Chem. Commun. 2003, 2682-2683.

[30] a) C. S. Consorti, G. Ebeling, F. R. Flores, F. Rominger, J. Dupont, Adv. Synth. Catal. 2004, 849 346, 617-624; b) G. D. Frey, J. Schütz, E. Herdtweck, W. A. Herrmann, Organometallics 2005,

[31] J. Dupont, C. S. Consorti, J. Spencer, Chem. Rev. 2005, 105, 2527-2572.

852

[32] V. P. W. Böhm, W. A. Herrmann, Chem. Eur. J. 2001, 7, 4191-4197.

[33] W. L. F. Armarego, Purification of Laboratory Chemicals, Eight ed., Butterworth Heinemann, Oxford, 2017. 
Scheme 1. The Mizoroki-Heck reaction.

860

861

Scheme 2. Synthesis of benzylphosphanes, phosphane-boranes and overall yields of the latter.

862

863

864

Scheme 3. Preparation of dimeric cyclometallated acetate-bridged complexes (not isolated) and the 865

Scheme 4. Mixture of complexes formed by reaction of $2 \mathrm{~b}$ with palladium acetate.

867

868

Scheme 5. Preparation of mononuclear complex 13c.

869

870

Scheme 6. Preparation of cationic complexes with a norbornadiene ligand.

871

872

Scheme 7. $\mathrm{M}-\mathrm{H}$ reaction between bromobenzene and butyl acrylate.

873

874

Figure.1 Catalytic precursors for the $\mathrm{M}-\mathrm{H}$ reaction developed by the groups of Herrmann,[7] Cole-

Hamilton[9] and those described in the present work.

876

877

Figure.2 Representation of the molecular structure of phosphane-boranes $2 \mathrm{c}^{\prime}$ (left) and $3 \mathrm{c}^{\prime}$ (right), with

878

ellipsoids shown at $50 \%$ probability level and hydrogen atoms omitted for clarity. Selected distances

879

880 $[\AA]$ and angles $\left({ }^{\circ}\right)$ : for $2 \mathrm{c}^{\prime} \mathrm{P}-\mathrm{C} 7,1.842(4), \mathrm{P}-\mathrm{C} 9,1.807(4), \mathrm{P}-\mathrm{C} 12,1.862(4), \mathrm{P}-\mathrm{B}, 1.890(5), \mathrm{C} 9-\mathrm{P}-\mathrm{C} 7$ 107.75(18), C7-P-C12 101.9(2), C9-P-C12 105.91(17), C7-P-B 113.8(2), C9-P-B 113.2(3), C12-PB 113.3(2). For 3c': P-C7, 1.821(3), P-C9, 1.842(3), P-C12, 1.859(3), P-B, 1.914(4), C9-P-C7 103.03(15), C7-P-C12 104.28(14), C9-P-C12 108.06(15), C7-P-B 110.72(16), C9-P-B 114.83(17), C12-P-B 114.77(17).

884

885

Figure.3. 31P $\{1 \mathrm{H}\}$ NMR spectrum of a mixture of brominated complexes obtained from $2 \mathrm{~b}$.

886

887

888

Figure.4 Figure 4. Molecular views of complexes 12a (left) and 12c (right) with ellipsoids drawn at 50 $\%$ probability level and $\mathrm{H}$ atoms removed for clarity. Selected distances $[\AA]$ and angles $\left(^{\circ}\right)$ for $12 \mathrm{a}$ : $\mathrm{Pd} 1-$ 889 C1 1.996(4); Pd1-Br1 2.5324(5); Pd1-P1 2.1930(11); Br1-Pd1-Br1 86.186(18); Br1-Pd1-C1 97.66 890 (11); C1-Pd1-P1 82.41(11); P1-Pd1-Br1 93.91(3). For 12c: Pd1-C1 2.0281(18); Pd1-Br1 2.5490(3); Pd1-P1 2.2007(11); Br1-Pd1-Br1 85.524(9); Br1-Pd1-C1 98.47 (5); C1-Pd1-P1 82.10(6); P1-Pd1- 
894 Figure.5 Figure 5. 1H NMR (CDCl3) spectrum of 15c.

895

896 Figure.6 Molecular view of complex 17 a with ellipsoids drawn at $50 \%$ probability level. $\mathrm{H}$ atoms and 897 the tetrafluoroborate anion have been removed for clarity. Selected distances $[\AA]$ and angles $\left({ }^{\circ}\right)$ : Pd1-P1 898 2.2561(6); Pd1-C8 2.020(2); Pd1-C1 2.300(2); Pd1-C2 2.275(2); Pd1-C6 2.301(3); Pd1-C7 2.316(2); 899 C1-C2 1.344(4); C6-C7 1.352(4); C8-Pd1-P1 79.56(7)

900

901 
903

904

905

$$
\mathrm{Ar}-\mathrm{X}+\boldsymbol{W}^{\mathrm{R}}
$$

906

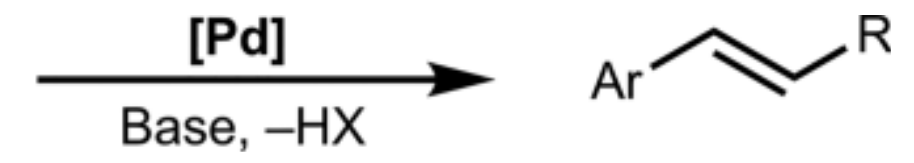




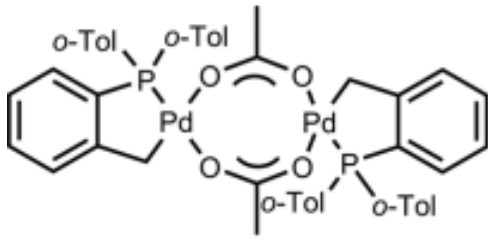

910

911<smiles>[R][R]1([R])Cc2ccccc2[P+](Br)(Br)P1([R])(Br)Br</smiles>

Cole-Hamilton et al., 2001

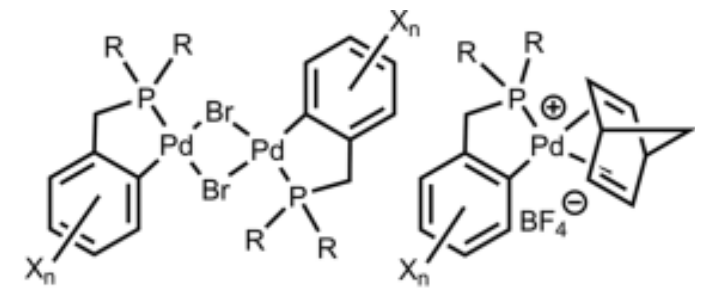

Present work 


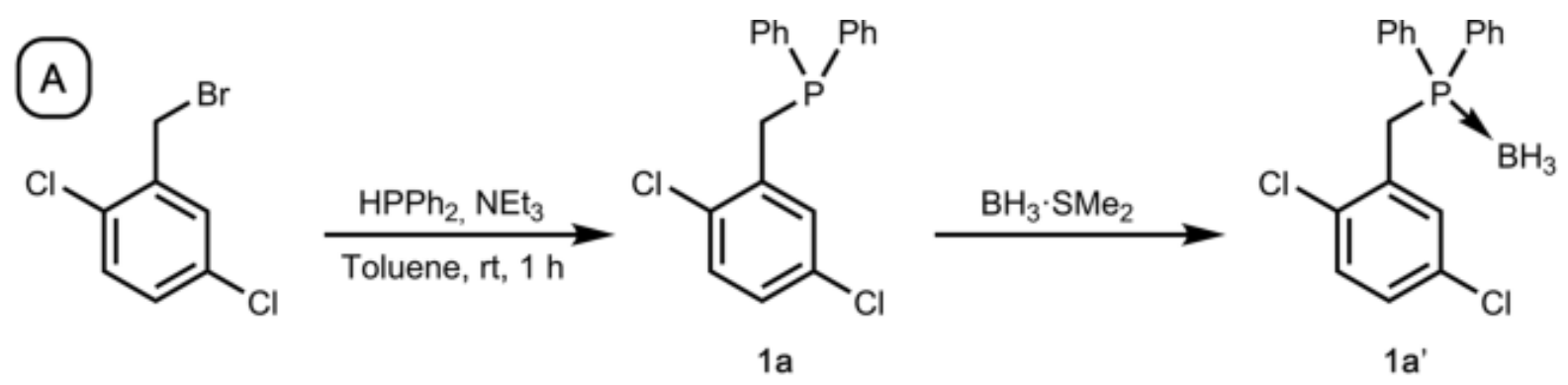<smiles>[X]c1ccc([X])c(CC(C)C)c1CBr</smiles>

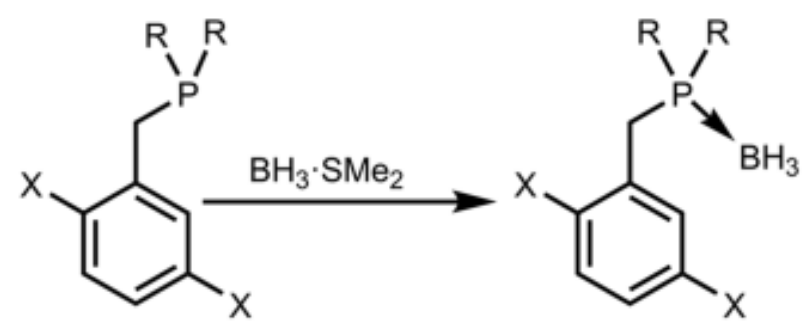
$\mathrm{X}=\mathrm{F}, \mathrm{Cl}$ $\mathrm{R}=\mathrm{Ph}(\mathrm{a}), \mathrm{Cy}(\mathrm{b}), i \operatorname{Pr}(\mathrm{c})$ $\mathrm{X}=\mathrm{Cl}$; 1a, 1b, 1c $\quad \mathrm{X}=\mathrm{Cl}$; 1a' (69\%), 1b' (49\%), 1c' $(68 \%)$ $X=F ; 2 a, 2 b, 2 c \quad X=F ; 2 a^{\prime}(36 \%), 2 b^{\prime}(68 \%), 2 c^{\prime}(61 \%)$

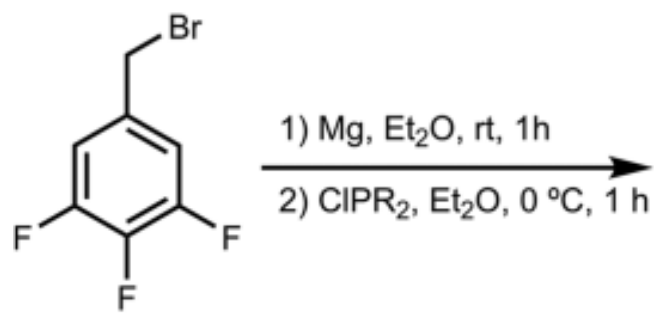<smiles>[R]P([R])Cc1cc(F)c(F)c(F)c1</smiles> 
918

919

920

921

922
FIGURE 2

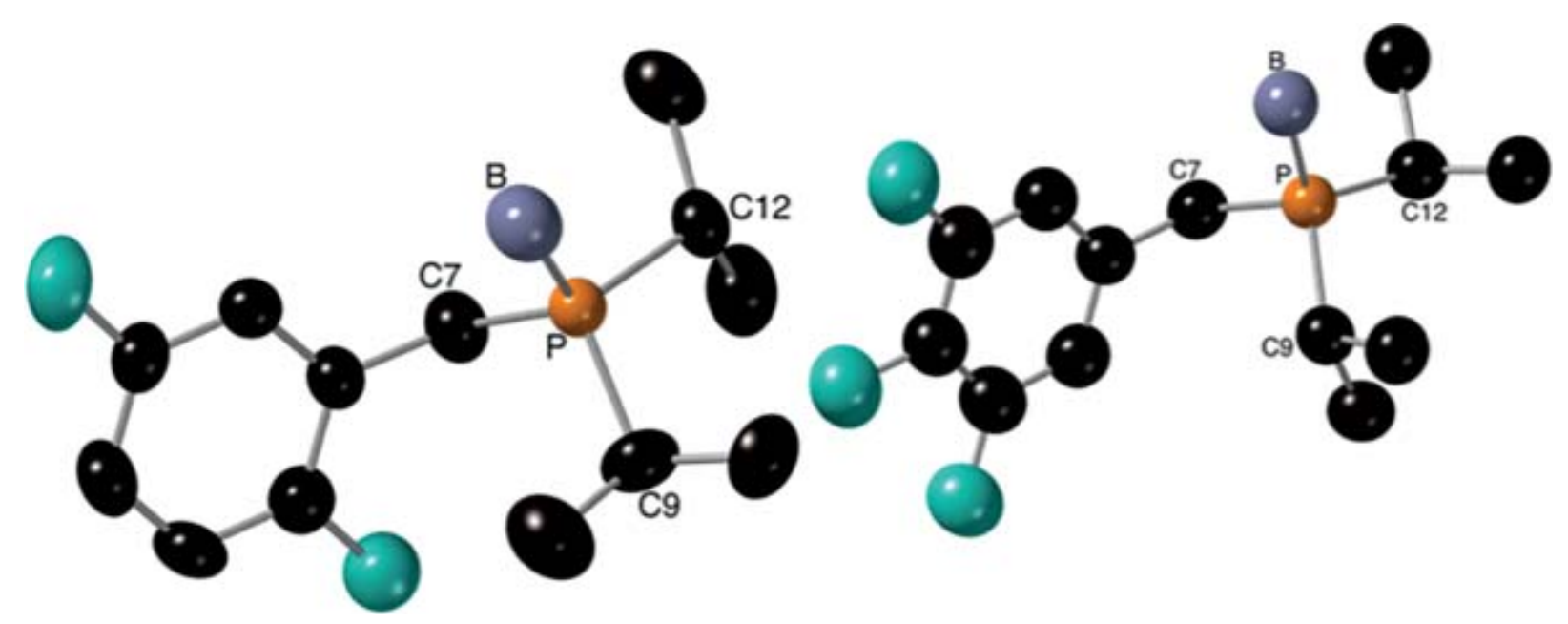


<smiles>[R][R]([O-])PCc1cc([X])ccc1[X]</smiles>

$\mathrm{X}=\mathrm{Cl} ; \mathbf{1 a}, \mathbf{1 b}, 1 \mathrm{c}$ $X=F ; 2 a, 2 b, 2 c$

$\mathrm{X}=\mathrm{H} ; \mathbf{4 a}, \mathbf{4 c}$

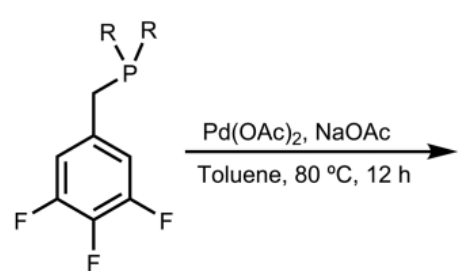

$3 a, 3 b, 3 c$

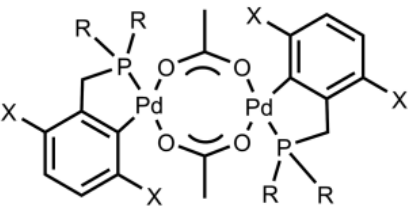

$\mathrm{X}=\mathrm{Cl} ; 5 \mathrm{a}, 5 \mathrm{~b}, \mathbf{5 c}$ $\mathrm{X}=\mathrm{F} ; \mathbf{6 a}, \mathbf{6 b}, \mathbf{6 c}$

$X=H ; 11 a, 11 c$

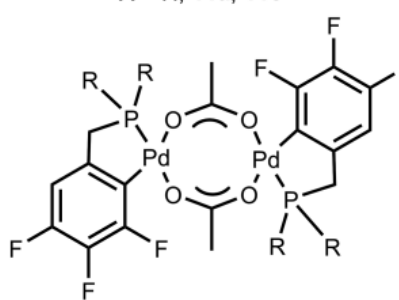

$7 a, 7 b, 7 c$

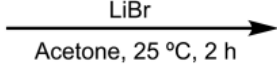<smiles>[X]c1ccc([X])c2c1CP([R])([R])([R7](Br)(Br)[P+]1(Br)c3c([X])ccc([X])c3C[PH]1([R])[R])[PH+]2[R]</smiles>

$X=\mathrm{Cl} ; \mathbf{8 a}(40 \%), \mathbf{8 b}(30 \%), \mathbf{8 c}(26 \%)$

$X=F ; 9 a(69 \%), 9 b(28 \%), 9 c(20 \%)$

$X=H ; 12 a(55 \%), 12 c(19 \%)$
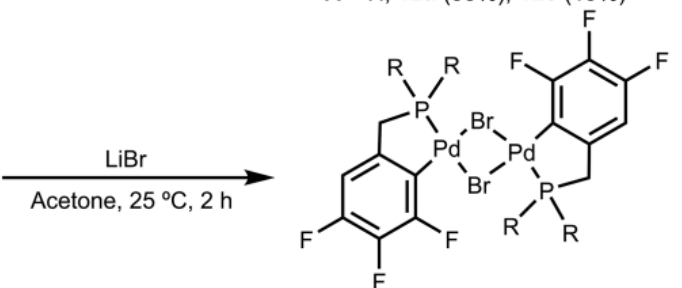

$10 \mathrm{a}(27 \%), 10 \mathrm{~b}(65 \%), 10 \mathrm{c}(34 \%)$ 


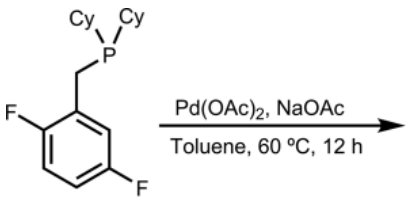

2b

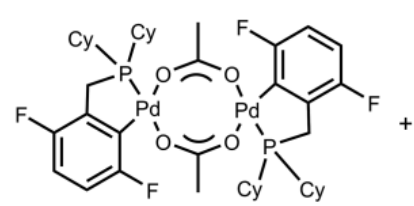

6b

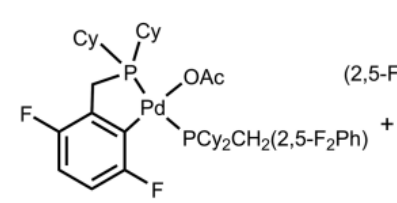

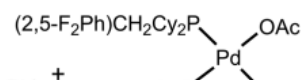

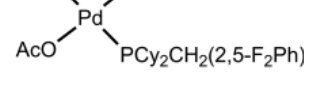
$6 b^{\prime}$

$\mathrm{LiBr}$, acetone

$25^{\circ} \mathrm{C}, 2 \mathrm{~h}$

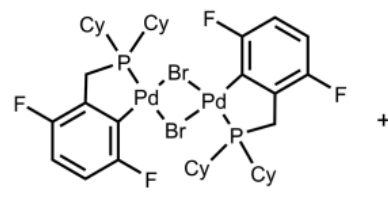

$9 b$

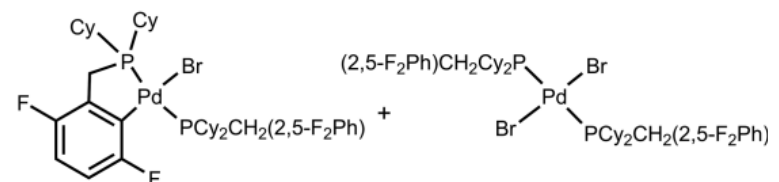

9b'

(3) 

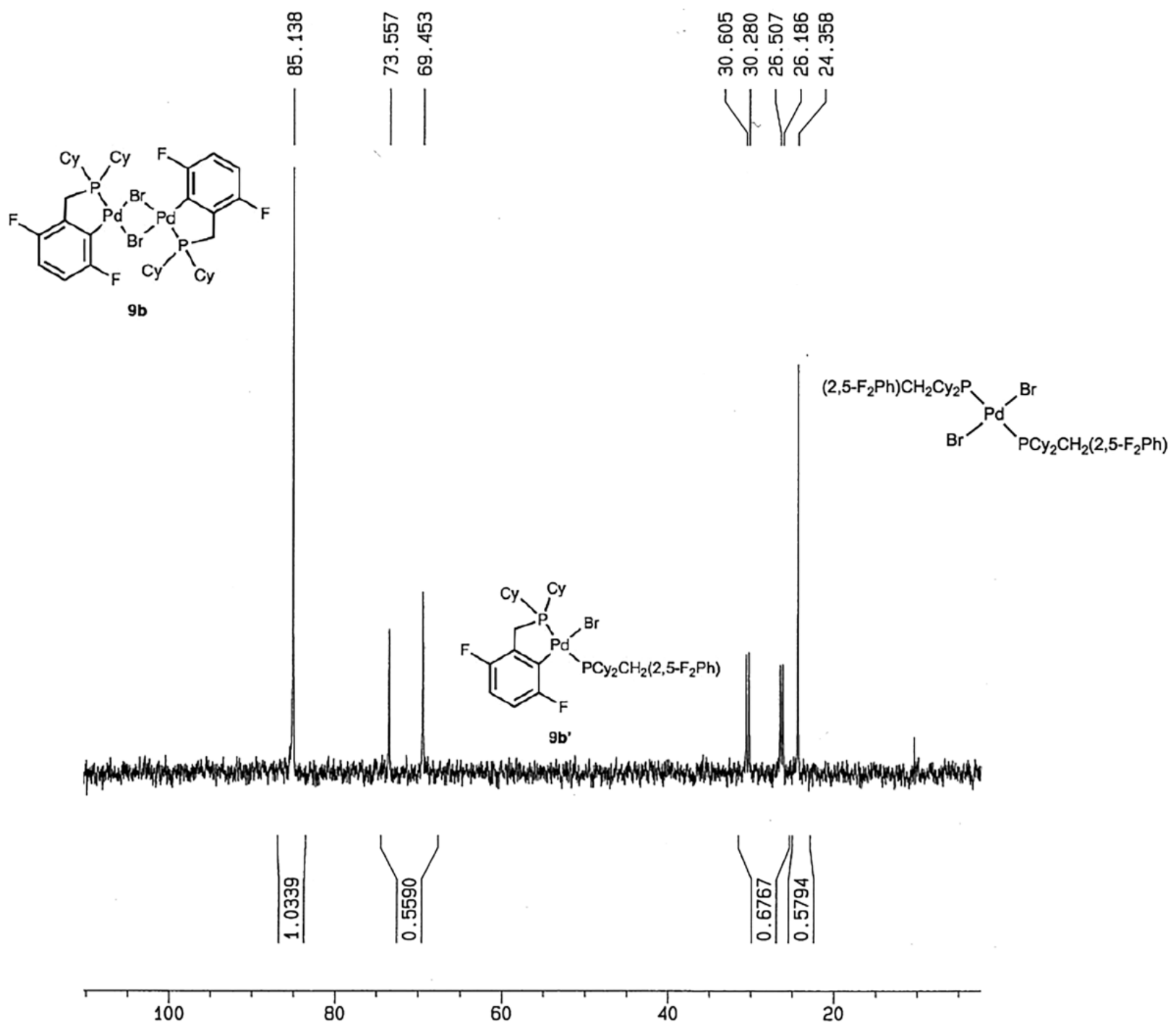
938

939

940

941

942
SCHEME 5

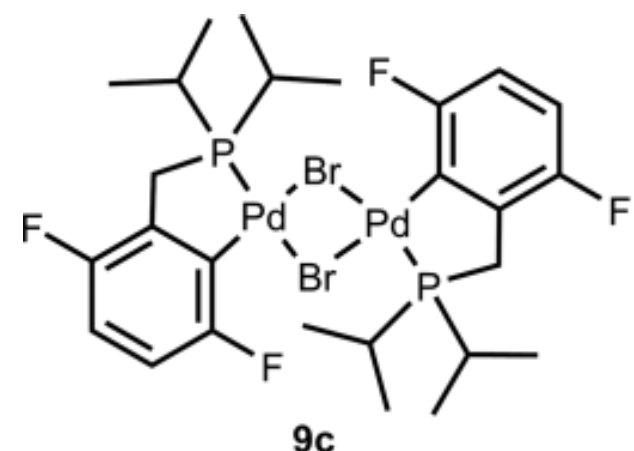

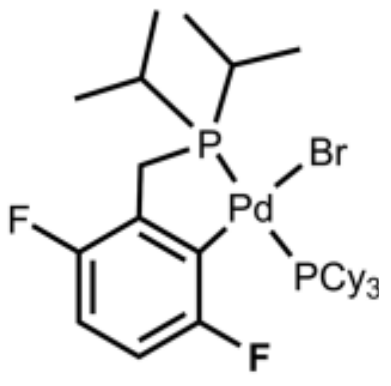

$13 c$ 
FIGURE 4

944

945

946
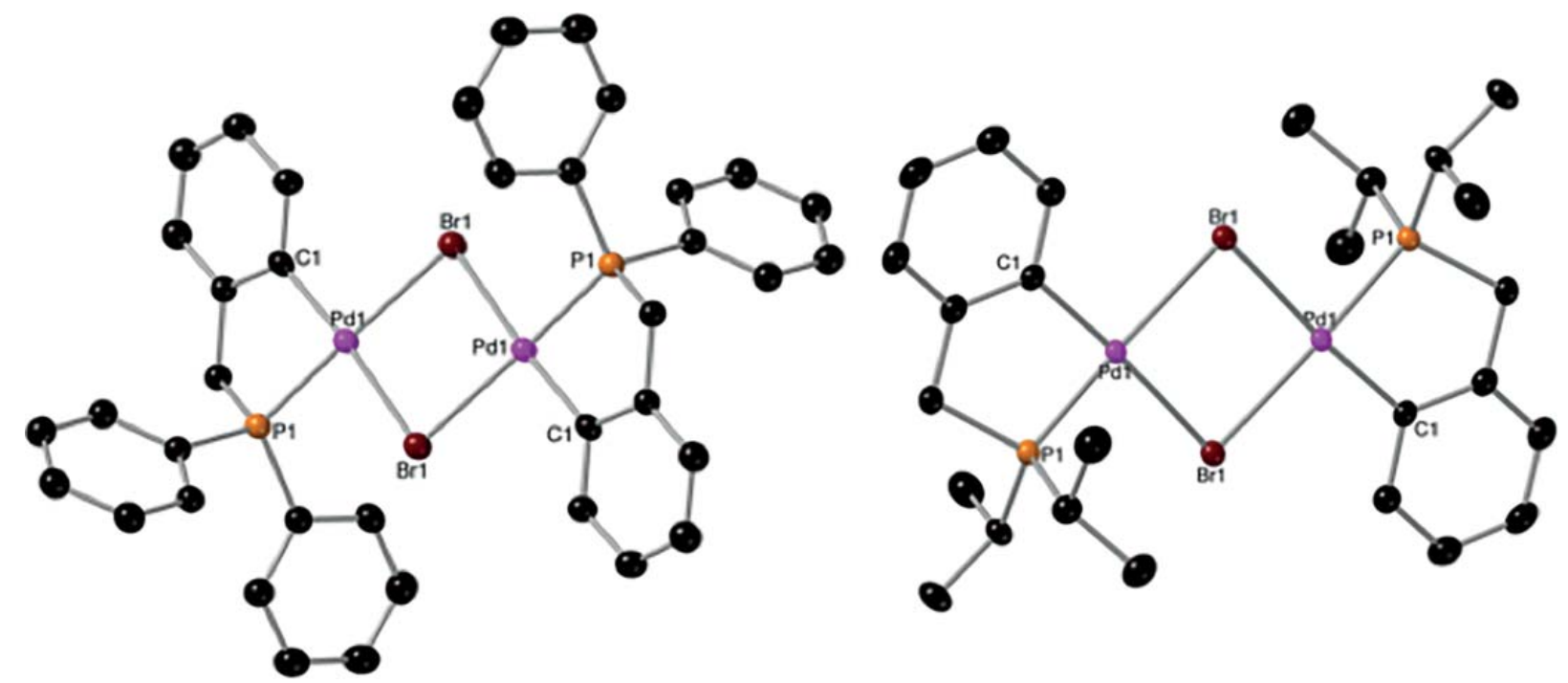

947 
<smiles>[X]c1ccc([X])c2c1CP([R])([R])([R])[R]2(Br)Br</smiles>

$8 c, 9 c, 12 a, 12 c$

$\mathrm{R}=\mathrm{Ph}(\mathbf{a}), i-\operatorname{Pr}(\mathbf{c})$

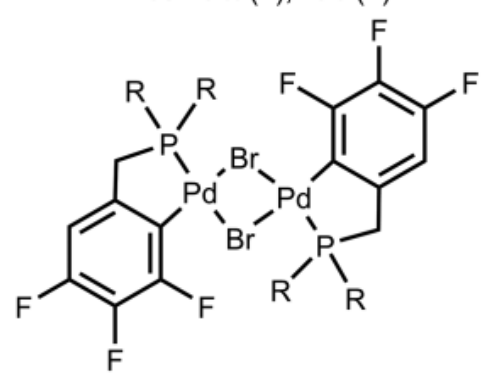

$10 \mathrm{a}, 10 \mathrm{~b}, 10 \mathrm{c}$

951

952 $\mathrm{R}=\mathrm{Ph}(\mathbf{a}), \mathrm{Cy}(\mathbf{b}), i-\operatorname{Pr}(\mathbf{c})$ $\longrightarrow \frac{\mathrm{AgBF}_{4}}{\mathrm{CH}_{2} \mathrm{Cl}_{2}, 25^{\circ} \mathrm{C}}$

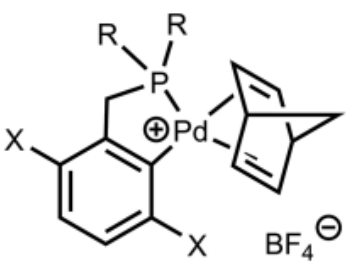

$\mathrm{R}=i-\mathrm{Pr}, \mathrm{X}=\mathrm{Cl}, 14 \mathrm{c} ; \mathrm{X}=\mathrm{F}, 15 \mathrm{c} ; \mathrm{X}=\mathrm{H}, 16 \mathrm{c}$ $\mathrm{R}=\mathrm{Ph}, \mathrm{X}=\mathrm{H}, 17 \mathrm{a}$

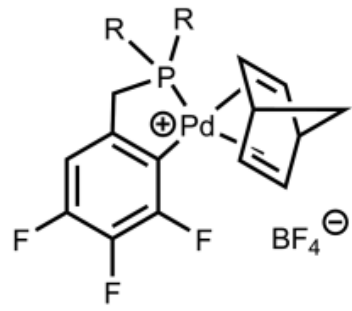

$18 a, 18 b, 18 c$ 


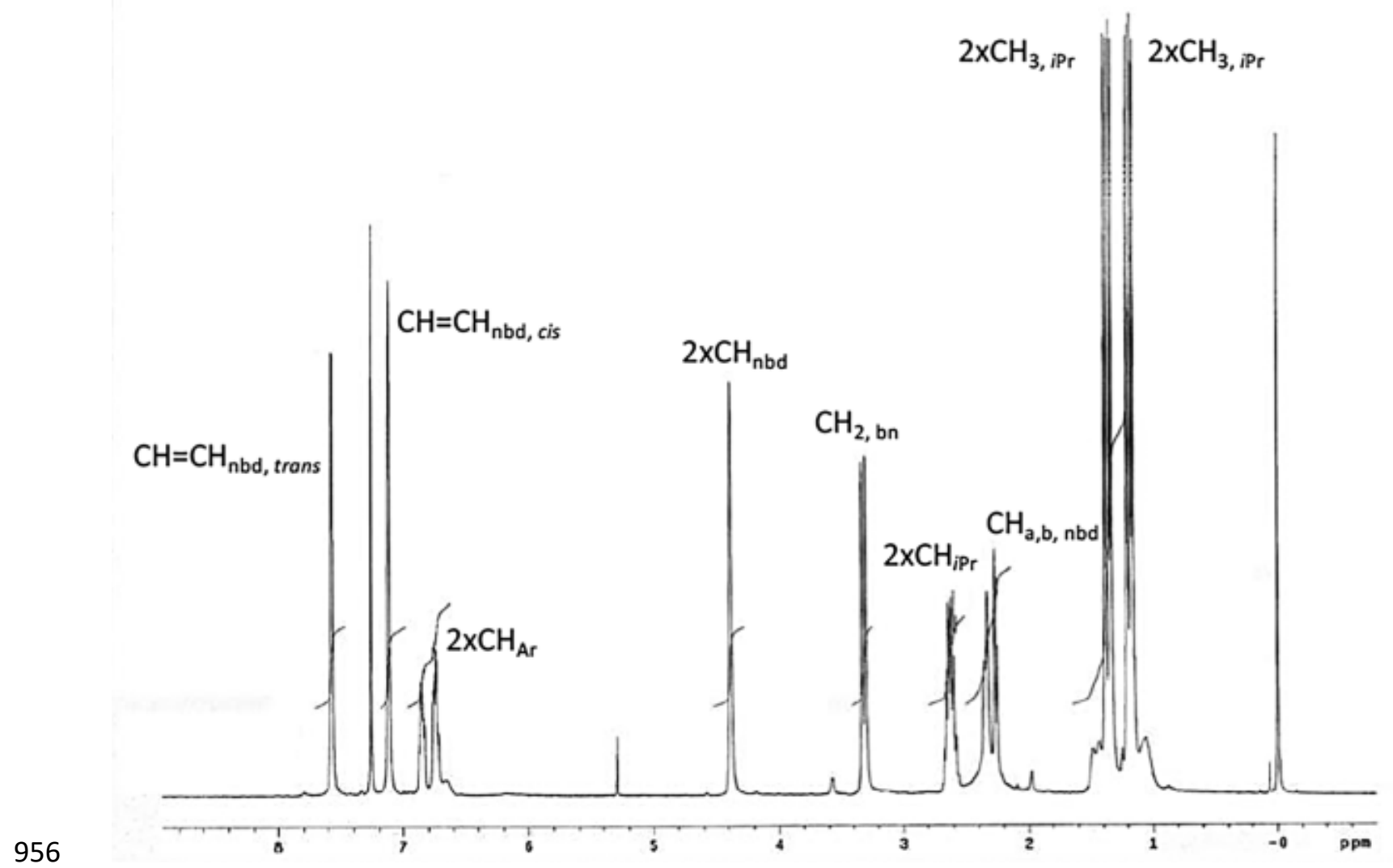




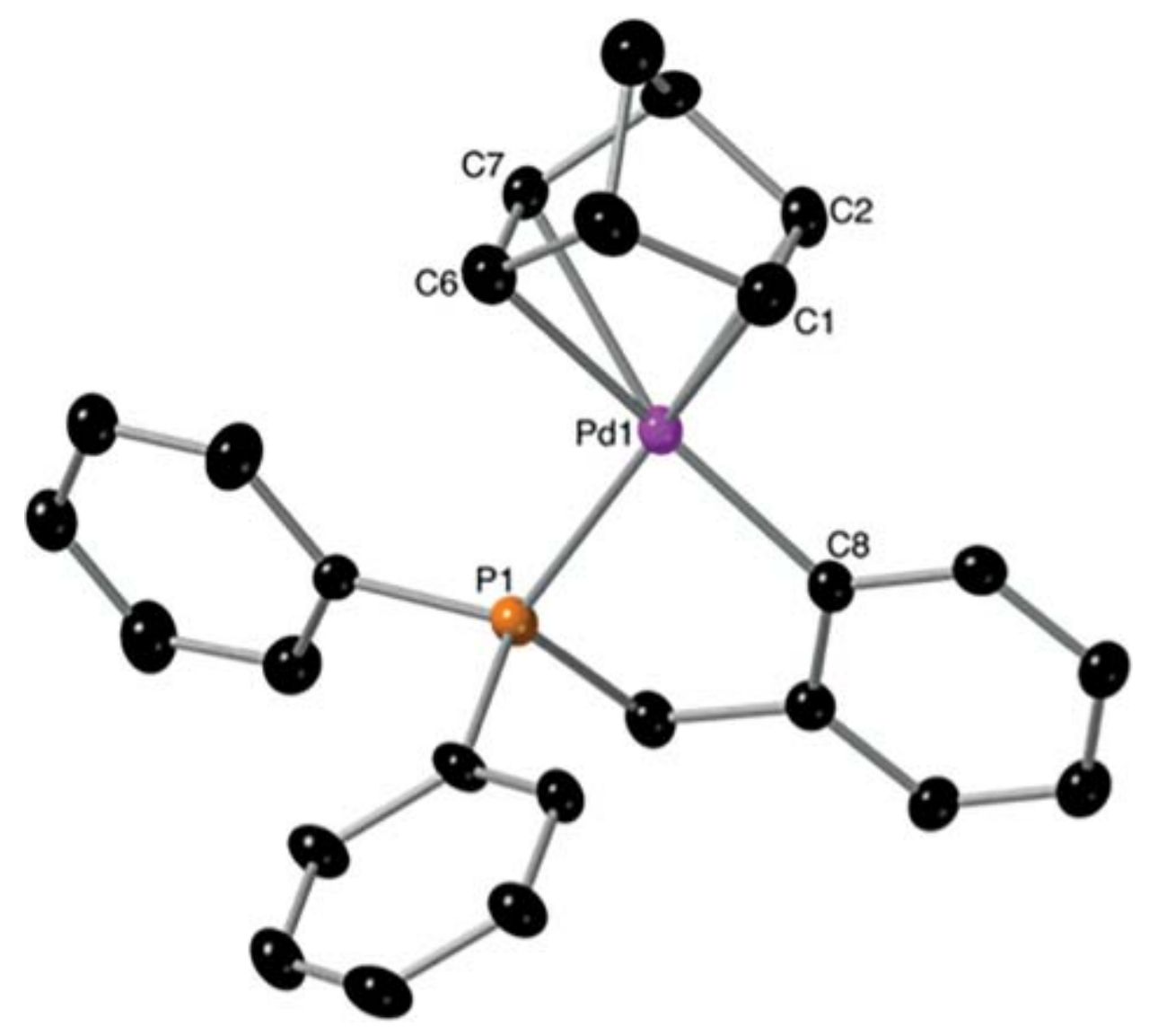


SCHEME 7

964

965

966

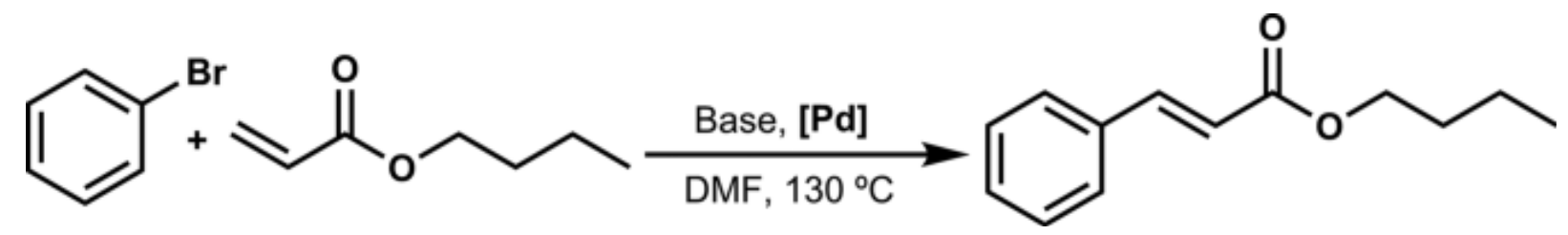

967 


\begin{tabular}{|c|c|c|c|c|}
\hline \multicolumn{2}{|c|}{$\begin{array}{l}\text { Fre phosphanes } \\
\qquad \begin{array}{c}d={ }^{31} \\
{\left[\mathrm{P}^{10} \mathrm{H}\right]}\end{array}\end{array}$} & Phosph & 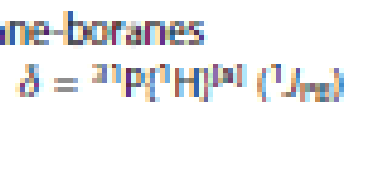 & 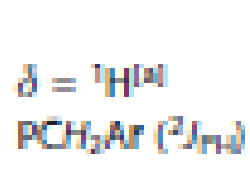 \\
\hline $1 \mathrm{a}=1$ & -12.8 & "la" & $+17.9[4.56]$ & $377 \mathrm{~d}, 12.0$ \\
\hline $1 b^{\mid ㄷ ㅗ ~}$ & +26 & $1 b^{\prime}$ & +29.7 (br) & 2.16 [d, $11.9 \rrbracket$ \\
\hline $1 d^{\mid-1}$ & +10.1 & $1 c$ & $+37.5[4.59)$ & 3.11 [d, 12.0 \\
\hline $2 a=1$ & $-11.5(\mathrm{~d}, 121)$ & $2 a^{\prime}$ & +17.7 (19.72] & 2.53 [0, 11.6$]$ \\
\hline $2 b^{\mid ㄷ ㅣ ~}$ & +24 (d, 95$]$ & $2 b^{\prime}$ & $+30.0[4,67)$ & $200[d, 11.2]$ \\
\hline $2 e^{\text {데 }}$ & $+10.6[0,10.5]$ & $2 c$ & $+36.1[4,56]$ & $200[0,11.2]$ \\
\hline $3 a$ & -9.9 & $3 a^{i}$ & $+17.2[4,63]$ & 250 [d, 11.6$]$ \\
\hline $3 b^{\mid ㄷ ㅣ ~}$ & +21 & $3 \mathbf{b}^{\prime}$ & $+27.7[\mathrm{q}, 59]$ & 294 [d, 11.6$)$ \\
\hline $3 d^{-11}$ & +10.6 & $3 e$ & $+34.6[4,51)$ & 295 [d, 11.6) \\
\hline $4 a \sqrt{17}$ & -10.0 & $4 a[15]$ & $+193[0,65]$ & 361 d. 1200 \\
\hline $4 c$ & +997 & - & - & - \\
\hline
\end{tabular}

971 Chemical shifts in ppm, coupling constants in Hz; 31P $\{1 \mathrm{H}\}(101.1 \mathrm{MHz}, 298 \mathrm{~K})$ and $1 \mathrm{H}(400 \mathrm{MHz}, 298$

972 K). Multiplicity and JPB and JHP in parenthesis. [a] Recorded in CDCl3; [b] Recorded in diethyl ether 973 with an external reference $(1 \% \mathrm{P}(\mathrm{OMe}) 3$ in C6D6); [c] Recorded in toluene with an external reference $974 \quad(1 \% \mathrm{P}(\mathrm{OMe}) 3$ in $\mathrm{C} 6 \mathrm{D} 6)$. 
Table 2. Selected NMR data of cyclopalladated complexes 5-12.[a]

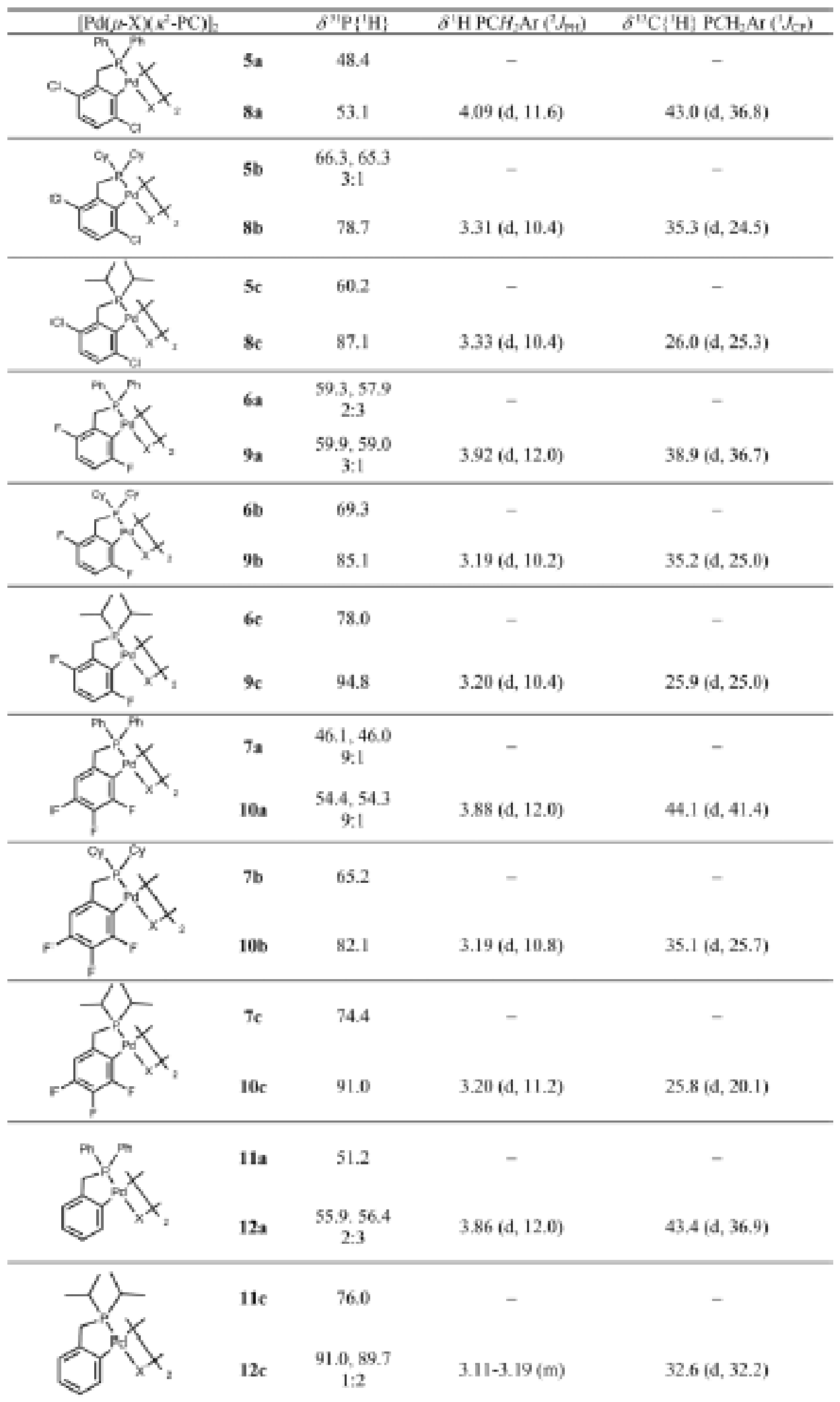

978 [a] Chemical shifts in ppm, coupling constants in Hz; 31P $\{1 \mathrm{H}\}(101.1 \mathrm{MHz}, 298 \mathrm{~K}), 1 \mathrm{H}(400 \mathrm{MHz}, 298$

$979 \mathrm{~K})$ and $13 \mathrm{C}\{1 \mathrm{H}\}(101 \mathrm{MHz}, 298 \mathrm{~K})$. Recorded toluene for $\mathrm{X}=\mathrm{OAc}$ and in $\mathrm{CDCl} 3$ for $\mathrm{X}=\mathrm{Br}$. 


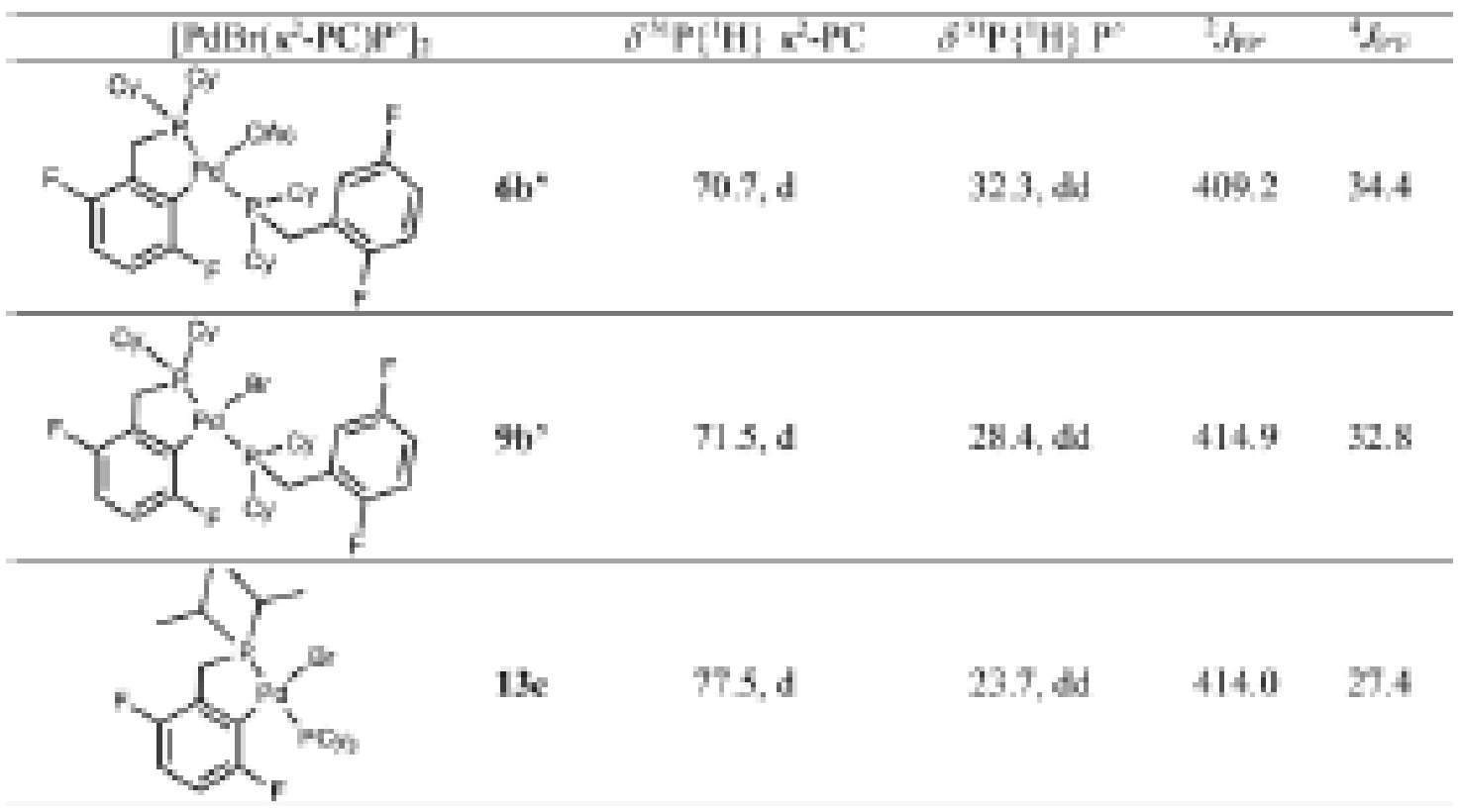

984 [a] Chemical shifts in ppm, coupling constants in $\mathrm{Hz} ; 31 \mathrm{P}\{1 \mathrm{H}\}(101.1 \mathrm{MHz}, 298 \mathrm{~K})$ and $1 \mathrm{H}(400 \mathrm{MHz}$, $985298 \mathrm{~K})$. 


\begin{tabular}{|c|c|c|c|c|c|c|}
\hline \multicolumn{2}{|l|}{$\left[\mathrm{Pd}\left(x^{2}-\mathrm{PC}\right)(\mathrm{nbd})\right] \mathrm{BF}_{4}$} & $\delta^{3} \mathrm{P}\{[\mathrm{H}\}$ & $\delta^{\prime}{ }_{\left({ }^{\prime} J_{\mathrm{HA}}\right)}^{\mathrm{PCH}}$ & $\begin{array}{c}\delta^{13} \mathrm{C}(\mathrm{H}) \mathrm{PC} H_{j} \mathrm{Ar} \\
\left(J_{O}\right)\end{array}$ & 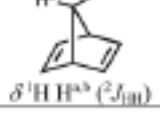 & $\begin{array}{c}\delta^{1} \mathrm{H} \mathrm{CH}-\mathrm{CH} \\
c i s, \text { twans }\end{array}$ \\
\hline & $14 \mathrm{e}$ & $86.6(\mathrm{~s})$ & 3.41 (bs) & 24.9 (d. 22.7) & $2.21(\mathrm{~m})$ & $\begin{array}{c}7.60-7.80 \\
\text { (bs) }\end{array}$ \\
\hline & $15 \mathrm{e}$ & $92.5(\mathrm{~s})$ & $3.31(d, 10.8)$ & $28.2(d, 34.1)$ & $\begin{array}{l}2.26(\mathrm{~d}, 8.8) \\
2.31 \text { (d:9.2) }\end{array}$ & $7.11,7.57$ \\
\hline & $16 \mathrm{e}$ & $95.5(\mathrm{~s})$ & $3.36(\mathrm{~d}, 10.8)$ & $31.3(\mathrm{~d}, 34.6)$ & $1.28(\mathrm{hr})$ & - \\
\hline & $17 a$ & $56.3(\mathrm{~s})$ & $4.22(d, 13.2)$ & $40.6(d, 39.1)$ & $1.92(\mathrm{br})$ & $6.74,7.08$ \\
\hline & $18 c$ & $88.7(\mathrm{~s})$ & $3.39(\mathrm{~d}, 11,6)$ & $41.2(d, 37.5)$ & $2.24-2.33(\mathrm{~m})$ & $7.06,7.55$ \\
\hline & $18 \mathrm{~b}$ & $81.3(\mathrm{~s})$ & $3.35(d, 11,4)$ & $33.6(d, 35.2)$ & $2.21(\mathrm{~m})$ & $6.95,7.46$ \\
\hline & $18 \mathrm{a}$ & $57.3(\mathrm{~s})$ & $4.15(d, 13,6)$ & 41.2 (d, 35.2) & $\begin{array}{c}2.09(\mathrm{hr}) \\
2.20(\mathrm{~d}, 9.2)\end{array}$ & $6.30,7.41$ \\
\hline
\end{tabular}

990 [a] Chemical shifts in ppm, coupling constants in $\mathrm{Hz} ; 31 \mathrm{P}\{1 \mathrm{H}\}(101.1 \mathrm{MHz}, 298 \mathrm{~K})$ and $1 \mathrm{H}(400 \mathrm{MHz}$, $991298 \mathrm{~K})$. 
Table 5. $\mathrm{M}-\mathrm{H}$ coupling reactions catalysed by cyclometallated $\mathrm{Pd}$ complexes.[a]

994

\begin{tabular}{|c|c|c|c|c|}
\hline Entry & Catalytic precursor & TOF $/ h^{-1}(1 \mathrm{~h})$ & TOF $/ h^{-1}(6 h)$ & Converslon/\% $/ 6 \mathrm{~h}$ ) \\
\hline 1 & $9 \mathrm{e}(2 \mathrm{~F}, \mathrm{IPT}$, dimeric) & 180 & 132 & 81 \\
\hline 2 & 12a $(2 \mathrm{H}, \mathrm{Ph}$, dimeric) & 307 & 149 & 90 \\
\hline 3 & $12 \mathrm{e}(2 \mathrm{H}, \mathrm{IPr}$, dimeric $)$ & 316 & 162 & 97 \\
\hline 4 & $14 \mathrm{c}(2 \mathrm{Cl}, \mathbb{P r}$, monomeric) & 250 & 90 & 55 \\
\hline 5 & $15 e(2 \mathrm{~F}, \mathrm{Pr}$, monomeric $)$ & 260 & 110 & 65 \\
\hline 6 & $16 \mathrm{e}(2 \mathrm{H}, \mathrm{IPr}$, monomenic) & 11 & 22 & 13 \\
\hline 7 & 17a $(\mathrm{ZH}, \mathrm{Ph}$, monomerlc) & 321 & 153 & 92 \\
\hline 8 & 18a (BF, Ph, monomeric) & 44 & 38 & 25 \\
\hline 9 & $18 \mathrm{~b}$ (3F, Cy, monomeric) & 107 & 28 & 17 \\
\hline 10 & $18 \mathrm{e}(3 \mathrm{~F}, \mathrm{PP}$, monomeric) & 160 & 60 & 34 \\
\hline $11^{[\mathrm{B}]}$ & $15 e(2 \mathrm{~F}, \mathbb{P r}$, dimeric) & 270 & 106 & 63 \\
\hline
\end{tabular}

996 a] Reaction conditions: bromobenzene $(10 \mathrm{mmol})$, butyl acrylate $(15 \mathrm{mmol}), \mathrm{Cs} 2 \mathrm{CO} 3$ (11 mmol), Pd

997 complex ( $0.1 \%$ of Pd with respect to bromobenzene) in $20 \mathrm{~mL}$ of DMF at $130{ }^{\circ} \mathrm{C}$. [b] Styrene instead of 998 butyl acrylate was used. 
1001

\begin{tabular}{|c|c|c|c|c|}
\hline Entry & Catalytic precursor & Aryl bromide & TOF/ $h^{-1}(\mathrm{l} h)$ & Conversion $\%$ \\
\hline 1 & $14 \mathrm{c}(2 \mathrm{Cl}$, iPr, monomeric $)$ & & 610 & $100(2 \mathrm{~h})$ \\
\hline 2 & $15 \mathrm{c}(2 \mathrm{~F}, \mathrm{iPr}$, mobomeric $)$ & & 850 & $100(2 \mathrm{~h})$ \\
\hline 3 & $18 \mathrm{c}(3 \mathrm{~F}, \mathrm{iPt}$, monomeric) & & 350 & $72(3 \mathrm{~h})$ \\
\hline 4 & $1 S_{c}(2 \mathrm{~F}, \mathrm{iPr}$, mosomeric) & & 145 & $52(6 \mathrm{~h})$ \\
\hline
\end{tabular}

1002

1003 [a] Reaction conditions: aryl bromide (10 mmol), butyl acrylate $(15 \mathrm{mmol}), \mathrm{Cs} 2 \mathrm{CO} 3$ (11 mmol), Pd 1004 complex $\left(0.1 \%\right.$ of Pd with respect to the aryl bromide) in $20 \mathrm{~mL}$ of DMF at $130{ }^{\circ} \mathrm{C}$. 